Preprint typeset in JHEP style - HYPER VERSION

$2004 / 04 / 05$

\title{
Instanton effects in quark form factor and quark-quark scattering at high energy
}

\author{
A.E. Dorokhov, I.O. Cherednikov \\ Joint Institute for Nuclear Research \\ RU-141980 BLTP JINR, Dubna, Russia \\ E-mail: dorokhov@thsun1.jinr.ru, igor.cherednikov@jinr.ru
}

\begin{abstract}
The nonperturbative effects in the high-energy processes involving strongly interacting particles are studied within the instanton liquid model of the QCD vacuum (ILM) by using the Wilson integral framework. The detailed analysis of nonperturbative contributions to the electromagnetic quark form factor is presented considering the structure of the instanton induced effects in the evolution equation describing the high energy behaviour of the form factor. It is shown that the instantons yield in high energy limit the logarithmic corrections to the amplitudes which are exponentiated in small instanton density parameter. By using the Gaussian interpolation of the constrained instanton solution, we show that the all-order multi-instanton contribution is well approximated by the weak field limit result. The role of the instantons in high energy diffractive quarkquark scattering, in particular, in formation of the soft Pomeron, is also considered. We show that within the ILM the $C$-odd diffractive amplitude is suppressed as $1 / s$ compared to the $C$-even one. The further applications of the developed approach in studying the nonperturbative effects in high energy hadronic processes are briefly discussed.
\end{abstract}

KeYwords: Instantons, Quark form factors, Soft Pomeron. 


\section{Contents}

1. Introduction 1

2. Electromagnetic quark form factor 3

3. Evolution equation for the quark form factor 6

4. Perturbative contributions to the Wilson integral 8

5. IR renormalon induced effects 11

6. Instanton induced contribution to the Wilson integral 14

7. Exponentiation of the instanton contributions in the dilute regime

8. Large- $q^{2}$ behaviour of the instanton contribution in the weak field approximation

9. All-order calculations of the Wilson loop for Gaussian profile 20

10. Instanton model of Pomeron 26

11. Conclusions 31

12. Acknowledgements 32

13. Appendix 32

\section{Introduction}

The powerful methods of the perturbative Quantum ChromoDynamics (pQCD) have been developed in order to describe the processes involving strongly interacting particles at high energies (for a review and comprehensive description of the methods, see, e.g., [1]). The total cross section of the $e^{+} e^{-}$annihilation and the logarithmic violation of scaling in deep inelastic scattering became the classical tests of pQCD already in the lowest orders of expansion in the strong coupling constant [2], and nowadays there are no doubts that the QCD Lagrangian provides a proper basis for a quantum field theory of strong interactions.

At the same time, the present status of pQCD does not allow one to consider it as the only tool for investigation of the hadronic properties even at highest energies accessible at modern machines. The perturbative methods should be supplied by certain information 
that can not be obtained directly from pQCD calculations. For instance, a nontrivial situation arises if, in order to make predictions of pQCD reliable, it is necessary to resum the soft part of the quark-gluon interaction to all orders. Moreover, in several situations the applicability of pQCD can be definitely justified only at asymptotically high energies, while in experimentally accessible region the nonperturbative effects are rather important and even dominant.

Meanwhile, the intermediate energy regime - where $Q^{2}$ is larger than the typical hadronic scale determined by $\Lambda_{Q C D}^{2}$ and lower than the characteristic scale of the chiral symmetry breaking: $\Lambda_{Q C D}^{2} \sim \Lambda_{\text {conf }}^{2}<Q^{2}<\Lambda_{\chi S B}^{2}$, is more convenient for detection of the nonperturbative phenomena. The complicated interplay of nonperturbative effects can lead in this regime to formation of the constituent quark which is, in a sense, an intermediate object between color-neutral hadron and pointlike structureless partons, associated with the fundamental QCD particles-quarks and gluons. Indeed, the form factors of constituent quarks were recently extracted [3] from the $J L a b$ experiment data [4] on the inelastic Nachtmann moments [5] of the unpolarized proton structure function $F_{2}^{p}\left(x, Q^{2}\right)$. And it was found that the size of a constituent quark determined from the data is about $0.2-0.3 \mathrm{fm}$ that corresponds to the mean instanton size in the instanton liquid model (ILM) [6, 7] which is also about $0.3 \mathrm{fm}$. In addition, the experimental and phenomenological investigations of the electromagnetic quark form factors at low and moderate energies can shed light on the problem of scaling violation in deep inelastic scattering processes.

The requirement of certain nonperturbative supplement for explicit perturbative calculations even in high energy domain as well the study of the hadron processes at low and moderate energies appear to be a natural ground for development of nonperturbative methods. Coming down in the energy scale more and more powers of the strong coupling constant has to be taken into account. Moreover, in the intermediate energy region the power corrections come into play with coefficients that are very sensitive to the intrinsic hadron structure. Typically, the coefficients of the expansion in powers of the coupling constant and the inverse momentum transfer squared are the hadronic matrix elements of quark-gluon operators normalized at low energy scale and have to be found by nonperturbative methods. The dependence of these matrix elements on the energy scale is governed by the evolution equations that are determined within $\mathrm{pQCD}$ for different hard processes. These equations start to be applicable at momentum transfer squared of order $1 \mathrm{GeV}^{2}$ or higher where the strong coupling constant becomes small. So, it is necessary to find the initial data for the evolution equation that is essentially a nonperturbative problem. The important nonperturbative effects in this energy region are treated by different approaches: QCD sum rules, lattice QCD, quark models, etc. Therefore, the study of the role of the nonperturbative input in investigation of the processes with strongly interacting particles is not only an interesting theoretical problem, but is an important task for phenomenology of hadronic physics.

It is naturally to relate the nonperturbative effects to the nontrivial structure of the QCD vacuum. In the last decades, a great progress has been made in study of the QCD ground state and a number of important results have been obtained that connect the properties of the vacuum with the hadron characteristics treating the QCD vacuum in the 
framework of the instanton liquid model [6, [7]. Considering QCD vacuum as an ensemble of instantons, one can describe a number of the low-energy phenomena in strong interactions on qualitative and quantitative levels [8, 9]. The importance of the instanton induced effects in the strong interaction is also supported by lattice simulations [8, 10]. The instanton picture is generally considered as a fruitful and perspective framework for hadronic physics. The role of instantons in the hard hadronic processes has been studied intensively, both theoretically and experimentally. The contact with the perturbative QCD results becomes possible providing the unique information about the quark-gluon distribution functions in the QCD vacuum and hadrons at low energy normalization point [11, 12, 13]. The perspectives for an unambiguous experimental detection of instanton effects are believed to be optimistic and promising.

Although the QCD vacuum is known to play an important role in the high-energy scattering processes, the direct investigation of these effects remains a difficult task. The idea that the nontrivial vacuum structure could be relevant in high energy hadronic processes was explicitly formulated in the context of the soft Pomeron problem in [14, 15, 16], and further developed using the eikonal approximation and the Wilson integral formalism in [17, 18].

In the present work, we report our recent results on investigation of the instanton induced effects in high energy regime for the electromagnetic (EM) quark form factor, i.e., the amplitude of the quark elastic scattering in an external color singlet gauge field. Then, we investigate the role of instantons in the diffractive quark-quark scattering and formation of the soft Pomeron. All the considered cases manifest a similar structure and are studied within the unified framework - the Wilson integral approach, which allows to study both perturbative and nonperturbative effects on the same ground. The method of path-ordered Wilson integrals is known as a powerful (and sometimes unique) tool in QCD which reformulates the theory in terms of the gauge invariant quantities - the Wilson loops - while the gauge fields are considered as chiral fields in the space of all possible loops [19]. The Green functions, amplitudes, and cross sections can be expressed completely in terms of the Wilson integrals over contours with geometry determined by specific kinematics in an intrinsically non-diagrammatic, (i.e., nonperturbative) fashion [20, 21, 22].

\section{Electromagnetic quark form factor}

The behavior of the hadronic form factors in various energy domains is one of the most important questions in the strong interaction phenomenology. The quark form factor has important phenomenological applications since it enters the cross sections of a number of high energy hadronic processes [2]. For example, the total cross section of the Drell-Yan process (normalized to the deep inelastic one) is determined by the ratio of the time-like and space-like form factors [23, 24]:

$$
\frac{\sigma_{n}^{D Y}}{\sigma_{n}^{P M}} \sim\left|\frac{F_{q}\left(Q^{2}\right)}{F_{q}\left(-Q^{2}\right)}\right|^{2},
$$

where $\sigma_{n}^{P M}$ is the $n$-th moment of the cross section calculated within the parton model. 
The electromagnetic quark form factors are determined via the elastic scattering amplitude of a quark in an external EM field:

$$
\mathcal{M}_{\mu}=F_{q}\left[\left(p_{1}-p_{2}\right)^{2}\right] \bar{u}\left(p_{1}\right) \gamma_{\mu} v\left(p_{2}\right)-G_{q}\left[\left(p_{1}-p_{2}\right)^{2}\right] \bar{u}\left(p_{1}\right) \frac{\sigma_{\mu \nu}\left(p_{1}-p_{2}\right)_{\nu}}{2 m} v\left(p_{2}\right)
$$

where $u\left(p_{1}\right), v\left(p_{2}\right)$ are the spinors of outgoing and incoming quarks, and $\sigma_{\mu \nu}=\left[\gamma_{\mu}, \gamma_{\nu}\right] / 2$. The kinematics of the process is described by two invariants (see Fig. 1a):

$$
s=\left(p_{1}+p_{2}\right)^{2}=2 m^{2}(1+\cosh \chi), \quad t=-Q^{2}=\left(p_{1}-p_{2}\right)^{2}=2 m^{2}(1-\cosh \chi),
$$

where $\chi$ is the scattering angle. In this work we assume that both the momentum transfer $-t$ and the total center-of-mass energy $s$ are large compared to the quark mass:

$$
\left(p_{1} p_{2}\right) \gg p_{1,2}^{2}=m^{2} \quad \text { or } \cosh \chi \gg 1 \text {. }
$$

In this regime the Pauli form factor $G_{q}$ is power suppressed and will be neglected. However, it should be emphasized that in low and moderate energy domains it becomes important and there arise interesting perturbative and nonperturbative effects (see, e.g., recent works [25, 26]).

The EM quark form factor is one of the simplest and convenient objects for investigation of the double logarithmic behaviour of the amplitudes in QCD in the high energy regime. From the methodological point of view, it requires a perturbative resummation procedure beyond the standard renormalization group techniques. Besides this, the resummation methods developed for this particular case can be applied to many other processes which possess the logarithmic enhancements near the kinematic boundaries. Similar resummation approach is also used in the study of the near-forward quark-quark scattering and the evaluation of the soft Pomeron properties [18]. In the latter case, the nonleading logarithmic terms become quite important.

The first example of large logarithm resummation was given in QED by V.V. Sudakov for the case of an off-shell fermion in the external Abelian gauge field in the doublelogarithmic approximation (DLA), where the terms of order of $\left(\alpha^{n} \ln ^{2 n} Q^{2}\right)$ are taken into account while the contributions from $O\left(\alpha^{n} \ln ^{2 n-1} Q^{2}\right)$ are neglected. The exponentiation of the leading double logarithmic result was found in [27]. In DLA the exponentiated form factor behaves as a rapidly decreasing at high momentum transfer function. This means that the elastic scattering of a quark by a virtual photon is strongly suppressed. The exponentiation for the on-shell form factor in the Abelian case was obtained in the DLA in [29] (the analytical calculation of the full electron vertex in two loops was performed in [28]).

These results eventually have been generalized for a more complicated non-Abelian gauge theory. First, the resumed DLA corrections in the QCD perturbative series were found to be consistent with the exponentiation in [30] (the inelastic on-shell form factor with emission of one and two gluons was calculated in the same context in [31]; the role of the quark Sudakov form factor in the description of $e^{+} e^{-}$one-photon annihilation in quarks and gluons was considered in DLA in [32]), and the all-order non-Abelian exponentiation has been proved in [33]. 
The question if the non-leading logarithmic terms could upset the DLA behaviour required a further work [34]. The all-(logarithmic)-order resummation was performed in the Abelian case and the exponentiation was demonstrated in [35]. In [36] the non-Abelian all-order exponentiation for the so-called hard part of the on-shell form factor has been shown within the powerful factorization approach ${ }^{1}$. However, the status of the soft part, containing all the infrared (IR) and collinear singularities and, as a consequence, all possible nonperturbative effects, remained unclear. The important results on the IR properties of the QCD quark form factors was obtained in [37] within the Wilson loop approach. In these works, the soft part of the form factor has been presented as the vacuum averaged exponent of the path integral of a gauge field over the contour of a special form - an angle with sides of semi-infinite length (Fig. 1a). The use of the gauge and renormalization groups invariance allowed to derive the perturbative evolution equation describing the high energy behaviour of the form factor taking into account all (power unsuppressed) parts of the factorized amplitude, both for the on- [38] and off-shell [39] cases. It was shown that the leading asymptotics is controlled by the cusp anomalous dimension which arises due to the multiplicative renormalization of the soft part, and can be calculated within the Wilson integral formalism up to the two-loop order [37]. Note that within the Wilson integral approach, the non-Abelian exponentiation can be proved independently [40, what is an important advantage of this framework.

The efficiency of the Wilson integral approach has been successfully demonstrated in a series of works [22, 41]. In these papers the non-diagrammatic framework was developed that allows to calculate the fermionic Green's functions, Sudakov form factors, amplitudes and cross sections in QED and QCD completely in terms of the world-line integrals, and thus avoid complicated diagrammatic factorization analysis.

The results presented above allow one to conclude that the leading high energy behaviour of the quark form factor in a non-Abelian gauge theory is completely determined by the perturbative evolution equation, and is given by the fast decreasing exponent:

$$
\sim \exp \left[-\frac{2 C_{F}}{\beta_{0}} \cdot \ln Q^{2} \ln \ln Q^{2}+O\left(\ln Q^{2}\right)\right],
$$

where

$$
C_{F}=\frac{N_{c}^{2}-1}{2 N_{c}}, \quad \beta_{0}=\frac{11 N_{c}-2 n_{f}}{3} .
$$

This rapid fall off is not changed by any other logarithmic contributions [36, 38, 39, 41]. However, the non-leading logarithmic corrections are nevertheless important for evaluation of the numerical value of the form factor at moderately large values of momentum transfer. Some of them are of a purely perturbative origin (e.g., sub-leading logarithmic terms), while the others can be attributed to the nonperturbative phenomena.

In the present work, we try to advocate the point of view that the true nonperturbative effects can be taken into account consistently in the evolution equation, and therefore they yield the non-vanishing subleading (perhaps, parameterically suppressed, but

\footnotetext{
${ }^{1}$ Note, that in this work the case where a time-like photon with large invariant mass decays into a quark-antiquark pair was considered, however it can be easily shown that the results remain true as well in the case of a quark scattering in an external EM field.
} 
still logarithmic) contributions $\sim \ln Q^{2}$ to the high energy behaviour of the amplitudes. Further, we analyze another possible source of contributions which can be considered as "nonperturbative" - the IR renormalon ambiguities (there are plenty of papers on this subject, for the most recent reviews see [42]). We demonstrate explicitly that they produce the corrections with different IR structure compared to that one generated by instantons. Moreover, as we show below these renormalon effects disappear in the dimensional regularization [24] and in the analytical perturbation theory [43], what means that they could be merely treated as artifacts of the incompleteness of the perturbative series resummation procedure.

In the following Sections we describe the consequences of the RG invariance of the factorized form factor and derive the linear evolution equation considering the nonperturbative input as the initial value for the perturbative evolution. We show that in the dilute phase the all-order single instanton contribution is exponentiated in small parameter of instanton density. Then, these nonperturbative effects are estimated in the weak-field approximation within the instanton model of QCD vacuum. By using the Gaussian simulation of the instanton profile function we show that the weak field result approximates well the all-order multi-instanton contribution. The large- $Q^{2}$ behaviour of the form factor is analyzed taking into account the leading perturbative and instanton induced contributions. The consequences of the IR renormalon ambiguities of the perturbative series and their relevance within the context of some analytization procedures are also studied. Finally, the Wilson integral techniques is applied to evaluation of the instanton contributions to the high-energy behavior of the $q q$-scattering amplitude in the Regge regime, that is the soft Pomeron problem.

\section{Evolution equation for the quark form factor}

The classification of the diagrams with respect to the momenta carried by their internal lines allows one to express the form factor $F_{q}$ in the amplitude (2.2) in the factorized form 35, 36, 38]

$$
F_{q}\left(q^{2}\right)=F_{H}\left(q^{2} / \mu^{2}, \alpha_{s}\right) \cdot F_{S}\left(q^{2} / m^{2}, \mu^{2} / \lambda^{2}, \alpha_{s}\right) \cdot F_{J}\left(\mu^{2} / \lambda^{2}, \alpha_{s}\right),
$$

where the hard, soft, and collinear (jet) part are separated by scale parameters $\mu^{2}, \lambda^{2}$. It is assumed the following relations between these scales $q^{2}>>\mu^{2}>>\lambda^{2}>1$. Note, that in the present paper all dimensional variables are assumed to be expressed in units of the

QCD scale $\Lambda_{Q C D}$, so that $q^{2}=Q^{2} / \Lambda_{Q C D}^{2}$, etc. The arbitrary scale $\mu^{2}$ is assumed to be equal to the UV normalization point.

Within the eikonal approximation the resummation of all logarithmic terms coming from the soft gluon subprocesses allows us to express $F_{S}$ in terms of the vacuum average of the gauge invariant path ordered Wilson integral [19, 44]

$$
F_{S}\left(q^{2} / m^{2}, \mu^{2} / \lambda^{2}, \alpha_{s}\right)=W\left(C_{\chi} ; \mu^{2} / \lambda^{2}, \alpha_{s}\right)=\frac{1}{N_{c}} \operatorname{Tr}\left\langle 0\left|\mathcal{P} \exp \left\{i g \int_{C_{\chi}} d x_{\mu} \hat{A}_{\mu}(x)\right\}\right| 0\right\rangle .
$$


In Eq. (3.2) the integration path corresponding to the considered process goes along the closed contour $C_{\chi}$ : the angle (cusp) with infinite sides (Fig. 1). We parameterize the integration path $C_{\chi}=\left\{z_{\mu}(t) ; t=[-\infty, \infty]\right\}$ as follows

$$
z_{\mu}(t)=\left\{\begin{array}{cc}
v_{1} t, & -\infty<t<0, \\
v_{2} t, & 0<t<\infty .
\end{array}\right.
$$

The gauge field $\hat{A}_{\mu}(x)=T^{a} A_{\mu}^{a}(x) \quad\left(\operatorname{Tr}\left[T^{a} T^{b}\right]=\frac{1}{2} \delta^{a b}\right) \quad$ belongs to the Lie algebra of the gauge group $S U\left(N_{c}\right)$, while the Wilson loop operator $\mathcal{P} \exp \left(i g \int d x A(x)\right)$ lies in its fundamental representation.

The Wilson integral (3.2) is multiplicatively renormalizable [37, 20]:

$$
W\left(C_{\chi} ; \mu^{2} / \lambda^{2}, \alpha_{s}\left(\mu^{2}\right)\right)=Z_{c u s p}\left(C_{\chi} ; \bar{\mu}^{2} / \mu^{2}, \alpha_{s}\left(\mu^{2}\right)\right) \cdot W_{\text {bare }}\left(C_{\chi} ; \bar{\mu}^{2} / \lambda^{2}, \alpha_{s}\left(\mu^{2}\right)\right),
$$

where $\bar{\mu}^{2}$ is the UV cutoff, $\mu^{2}$ is the normalization point, and $\lambda^{2}$ is the IR cutoff. The presence of the IR divergence in (3.4) is a common feature of $o n$-shell amplitudes in massless QCD. Therefore, we can define the cusp anomalous dimension $\Gamma_{\text {cusp }}$ :

$$
\begin{aligned}
\frac{1}{2} \Gamma_{c u s p}\left(C_{\chi} ; \alpha_{s}\left(\mu^{2}\right)\right) & =-\mu^{2} \frac{d}{d \mu^{2}} \ln W\left(C_{\chi} ; \mu^{2} / \lambda^{2}, \alpha_{s}\left(\mu^{2}\right)\right)= \\
& =-\mu^{2} \frac{d}{d \mu^{2}} \ln Z_{c u s p}\left(C_{\chi} ; \bar{\mu}^{2} / \mu^{2}, \alpha_{s}\left(\mu^{2}\right)\right) .
\end{aligned}
$$

It can be shown that the cusp anomalous dimension (3.5) is linear in the scattering angle $\chi$ to all orders of perturbation theory in the large- $q^{2}$ regime 37]:

$$
\Gamma_{\text {cusp }}\left(C_{\chi} ; \alpha_{s}\right)=\ln q^{2} \Gamma_{c u s p}\left(\alpha_{s}\right)+O\left(\ln ^{0} q^{2}\right)
$$

where

$$
\Gamma_{\text {cusp }}\left(\alpha_{s}\right)=\sum_{0}^{\infty}\left(\frac{\alpha_{s}}{\pi}\right)^{n} C_{n} A_{n},
$$

$C_{n}$ are maximally non-Abelian color factors $\left(C_{n}=C_{F} N_{c}^{n-1}\right.$ in lowest orders), and $A_{n}$ are some numerical factors. The observation (3.6) is crucial for the efficiency of the analysis of high energy behavior within the Wilson integral formalism, since calculating the cusp anomalous dimension only in leading (one-loop) order one obtains the dominant contribution to the high energy asymptotics.

The total form factor $F_{q}$ is renormalization invariant quantity and satisfies to the renorm group ( $\mathrm{RG})$ equation:

$$
\mu^{2} \frac{d}{d \mu^{2}} F_{q}\left(\mu^{2}, \alpha_{s}\left(\mu^{2}\right)\right)=\left(\mu^{2} \frac{\partial}{\partial \mu^{2}}+\beta\left(\alpha_{s}\right) \frac{\partial}{\partial \alpha_{s}}\right) F_{q}\left(\mu^{2}, \alpha_{s}\left(\mu^{2}\right)\right)=0
$$

which in the large- $q^{2}$ regime leads to the following relations

$$
\mu^{2} \frac{d}{d \mu^{2}}\left[\frac{\partial \ln F_{H}}{\partial \ln q^{2}}\right]=-\mu^{2} \frac{d}{d \mu^{2}}\left[\frac{\partial \ln F_{S}}{\partial \ln q^{2}}\right]=\frac{1}{2} \Gamma_{c u s p}\left(\alpha_{s}\right) .
$$


To avoid the problems with additional light-cone singularities in the soft part, we work with logarithmic derivatives in $q^{2}$ [38, 45]. The collinear part $F_{J}$ being independent on $q^{2}$ does not contribute to these equations.

Then, from the Eqs. (3.9, 3.5, 3.6) one finds after simple calculations [38]:

$$
\begin{gathered}
\frac{\partial \ln F_{H}\left(q^{2}\right)}{\partial \ln q^{2}}=\int_{q^{2}}^{\mu^{2}} \frac{d \xi}{2 \xi} \Gamma_{c u s p}\left(\alpha_{s}(\xi)\right)+\Gamma\left(\alpha_{s}\left(q^{2}\right)\right), \\
\frac{\partial \ln F_{S}\left(q^{2}\right)}{\partial \ln q^{2}}=-\int_{\lambda^{2}}^{\mu^{2}} \frac{d \xi}{2 \xi} \Gamma_{c u s p}\left(\alpha_{s}(\xi)\right)+\frac{\partial \ln W_{n p}\left(q^{2}\right)}{\partial \ln q^{2}},
\end{gathered}
$$

where the "integration constant" of the hard part reads

$$
\Gamma\left(\alpha_{s}\left(q^{2}\right)\right)=\left.\frac{\partial \ln F_{H}\left(q^{2}\right)}{\partial \ln q^{2}}\right|_{\mu^{2}=q^{2}},
$$

and $W_{n p}$ arises as the initial value of the soft part:

$$
\frac{\partial \ln W_{n p}\left(q^{2}\right)}{\partial \ln q^{2}}=\left.\frac{\partial \ln F_{S}\left(q^{2}\right)}{\partial \ln q^{2}}\right|_{\mu^{2}=\lambda^{2}},
$$

and is the only quantity where, according to our suggestion, the nonperturbative effects take place [46, 47].

Then we get the $q^{2}$-dependence of the total form factor at large $q^{2}$ :

$$
\begin{gathered}
\ln \frac{F_{q}\left(q^{2}\right)}{F_{q}\left(q_{0}^{2}\right)}= \\
=-\int_{q_{0}^{2}}^{q^{2}} \frac{d \xi}{2 \xi}\left[\ln \frac{q^{2}}{\xi} \Gamma_{c u s p}\left(\alpha_{s}(\xi)\right)-2 \Gamma\left(\alpha_{s}(\xi)\right)\right]-\ln \frac{q^{2}}{q_{0}^{2}} \int_{\lambda^{2}}^{q_{0}^{2}} \frac{d \xi}{2 \xi} \Gamma_{c u s p}\left(\alpha_{s}(\xi)\right)+\ln \frac{W_{n p}\left(q^{2}\right)}{W_{n p}\left(q_{0}^{2}\right)} .
\end{gathered}
$$

In the next subsection, we explicitly calculate the perturbative quantities entering Eq. (3.14) in the one loop perturbative QCD approximation and in the other parts we concentrate on the nonperturbative part within ILM.

\section{Perturbative contributions to the Wilson integral}

The analysis of the hard contributions [36, 38] at large $q^{2}$ yields:

$$
\frac{\partial \ln F_{H}\left(q^{2} / \mu^{2}, \alpha_{s}\right)}{\partial \ln q^{2}}=-\frac{\alpha_{s}}{2 \pi} C_{F}\left(\ln \frac{q^{2}}{\mu^{2}}-\frac{3}{2}\right)+O\left(\alpha_{s}^{2}\right) .
$$

For the hard "integration constant" (3.12) one has:

$$
\Gamma\left(\alpha_{s}\left(q^{2}\right)\right)=\frac{3}{4} \frac{\alpha_{s}\left(q^{2}\right)}{\pi} C_{F} .
$$


The expression (4.1) is IR-safe, while the low-energy information is accumulated in the soft part of the quark form factor $F_{S}$. The Wilson integral (3.2) can be presented as a series:

$$
\begin{gathered}
W\left(C_{\chi}\right)=1+\frac{1}{N_{c}} \operatorname{Tr}\langle 0| \sum_{n=2}(i g)^{n} \int_{C_{\chi}} \int_{C_{\chi}} \ldots \int_{C_{\chi}} d x_{\mu_{n}}^{n} d x_{\mu_{n-1}}^{n-1} \ldots d x_{\mu_{1}}^{1} . \\
\cdot \theta\left(x^{n}, x^{n-1}, \ldots, x^{1}\right) \hat{A}_{\mu_{n}}\left(x^{n}\right) \hat{A}_{\mu_{n-1}}\left(x^{n-1}\right) \ldots \hat{A}_{\mu_{1}}\left(x^{1}\right)|0\rangle,
\end{gathered}
$$

where the function $\theta(x)$ orders the color matrices along the integration contour.

The leading order of the expansion (4.3) is given by expression (see Fig. 1b-c):

$$
\begin{aligned}
W_{\text {bare }}^{(1)}\left(C_{\chi}\right) & =-\frac{g^{2}}{2} \frac{1}{N_{c}} \operatorname{Tr}\left(T^{a} T^{b}\right) \int_{C_{\chi}} d x_{\mu} \int_{C_{\chi}} d y_{\nu} \mathcal{D}_{\mu \nu}^{a b}(x-y) \\
& =-\frac{g^{2}}{2} \frac{1}{2 N_{c}} \int_{C_{\chi}} d x_{\mu} \int_{C_{\chi}} d y_{\nu} \mathcal{D}_{\mu \nu}(x-y)
\end{aligned}
$$

where the gauge field propagator $\mathcal{D}_{\mu \nu}(z)$ in $n$-dimensional space-time $(n=4-2 \varepsilon)$ reads:

$$
\mathcal{D}_{\mu \nu}(z)=\left\langle 0\left|\mathcal{T} A_{\mu}^{\bar{a}}(z) A_{\nu}^{\bar{a}}(0)\right| 0\right\rangle
$$

The exponentiation theorem for non-Abelian path-ordered Wilson integrals 40] allows us to express (to one-loop accuracy) the Wilson integral (3.2) as the exponentiated one-loop term of the series (4.3):

$$
W_{\text {bare }}\left(C_{\chi} ; \varepsilon, \bar{\mu}^{2} / \lambda^{2}\right)=\exp \left[W_{\text {bare }}^{(1)}\left(C_{\chi} ; \varepsilon, \bar{\mu}^{2} / \lambda^{2}\right)+O\left(\alpha_{s}^{2}\right)\right] .
$$

In general, the expression (4.4) contains UV and IR divergences, that can be multiplicatively renormalized in a consistent way [20]. In the present work, we use the dimensional regularization for the UV singularities, and define the "gluon mass" $\lambda^{2}$ as the IR regulator.

The dimensionally regularized free propagator in covariant gauge reads $(n=4-$ $2 \varepsilon), \varepsilon>0$ :

$$
\begin{gathered}
\mathcal{D}_{\mu \nu}(z ; \xi)=\left(N_{c}^{2}-1\right) D_{\mu \nu}(z ; \xi) \\
D_{\mu \nu}(z ; \xi)=\mu^{4-n} \frac{1}{i} \int \frac{d^{n} k}{(2 \pi)^{n}} \mathrm{e}^{-i k z}\left(\frac{g_{\mu \nu}}{k^{2}-\lambda^{2}+i 0}-\xi \frac{k_{\mu} k_{\nu} /\left[k^{2}-(1-\xi) \lambda^{2}+i 0\right]}{k^{2}-\lambda^{2}+i 0}\right),
\end{gathered}
$$

where $\xi$ is a gauge fixing parameter. It is convenient to use the representation

$$
D_{\mu \nu}(z)=g_{\mu \nu} \partial_{z}^{2} \Delta_{1}\left(\varepsilon, z^{2}, \bar{\mu}^{2} / \lambda^{2}\right)-\partial_{\mu} \partial_{\nu} \Delta_{2}\left(\varepsilon, z^{2}, \bar{\mu}^{2} / \lambda^{2}\right),
$$

where $\bar{\mu}^{2}$ is a parameter of dimensional regularization.

By using integrals (13.1) - (13.6) from Appendix A, the dimensionally regularized formula for the leading order (LO) term (4.4) can be written as 46.

$$
W_{\text {bare }}^{(1)}\left(C_{\chi} ; \varepsilon, \bar{\mu}^{2} / \lambda^{2}, \alpha_{s}\right)=8 \pi \alpha_{s} C_{F} h(\chi)(1-\varepsilon) \Delta_{1}\left(\varepsilon, 0, \bar{\mu}^{2} / \lambda^{2}\right),
$$

where $h(\chi)$ is the universal cusp factor:

$$
h(\chi)=\chi \operatorname{coth} \chi-1
$$


which at large- $q^{2}$ is given by:

$$
\lim _{\chi \rightarrow \infty} h(\chi) \rightarrow \chi \propto \ln \frac{q^{2}}{m^{2}}
$$

In Eq. (4.9), for the perturbative gauge field one has

$$
\Delta_{1}\left(\varepsilon, 0, \bar{\mu}^{2} / \lambda^{2}\right)=-\frac{1}{16 \pi^{2}}\left(4 \pi \frac{\bar{\mu}^{2}}{\lambda^{2}}\right)^{\varepsilon} \frac{\Gamma(\varepsilon)}{1-\varepsilon} .
$$

The independence of the expression (4.9) of the function $\Delta_{2}$ is a direct consequence of the gauge invariance.

Thus, in the one-loop approximation one gets

$$
W_{\text {bare }}\left(C_{\chi} ; \varepsilon, \bar{\mu}^{2} / \lambda^{2}, \alpha_{s}\right)=1-\frac{\alpha_{s}}{2 \pi} C_{F} h(\chi)\left(\frac{1}{\varepsilon}-\gamma_{E}+\ln 4 \pi+\ln \frac{\bar{\mu}^{2}}{\lambda^{2}}\right),
$$

and the cusp dependent renormalization constant, within the $\overline{M S}$-scheme which fixes the UV normalization point, reads:

$$
Z_{\text {cusp }}\left(C_{\chi} ; \varepsilon, \bar{\mu}^{2} / \mu^{2}, \alpha_{s}\left(\mu^{2}\right)\right)=1+\frac{\alpha_{s}\left(\mu^{2}\right)}{2 \pi} C_{F} h(\chi)\left(\frac{1}{\varepsilon}-\gamma_{E}+\ln 4 \pi\right)+O\left(\alpha_{s}^{2}\right) .
$$

Using the Eq. (4.9), one finds the known one-loop result for the perturbative field, which contains the dependence on the UV normalization point $\mu^{2}$ and IR cutoff $\lambda^{2}$ (see e.g., [37, 41]):

$$
W_{p t}^{(1)}\left(C_{\chi} ; \mu^{2} / \lambda^{2}, \alpha_{s}\left(\mu^{2}\right)\right)=-\frac{\alpha_{s}\left(\mu^{2}\right)}{2 \pi} C_{F} h(\chi) \ln \frac{\mu^{2}}{\lambda^{2}}+O\left(\alpha_{s}^{2}\right) .
$$

Therefore, in the leading order the kinematic dependence of the expression (4.4) is factorized into the cusp factor $h(\chi)$ 44.

From the one-loop result (4.15), the cusp anomalous dimension which satisfies the RG equation $(3.5)$ in one-loop order is given by ${ }^{2}$ :

$$
\Gamma_{\text {cusp }}^{(1)}\left(\alpha_{s}\left(\mu^{2}\right)\right)=\frac{\alpha_{s}\left(\mu^{2}\right)}{\pi} C_{F} .
$$

Substituting into the Eq. (3.14) the anomalous dimension (4.16) with the strong coupling constant given in the one-loop approximation, one finds

$$
\begin{gathered}
F_{q}^{(1)}\left(q^{2}\right)= \\
\exp \left[-\frac{2 C_{F}}{\beta_{0}}\left[\ln q^{2}\left(\ln \frac{\ln q^{2}}{\ln \lambda^{2}}-1\right)-\frac{3}{2} \ln \frac{\ln q^{2}}{\ln q_{0}^{2}}+\ln q_{0}^{2}\left(1-\ln \frac{\ln q_{0}^{2}}{\ln \lambda^{2}}\right)\right]+W_{n p}\left(q^{2}\right)\right] F^{(1)}\left(q_{0}^{2}\right) .
\end{gathered}
$$

Note, that the exponent in Eq. (4.17) has an unphysical singularity at $\lambda^{2}=1$ (in dimensional notations, $\left.\bar{\lambda}^{2}=\Lambda_{Q C D}^{2}\right)$, i. e., where the one-loop coupling constant $\alpha_{s}\left(\bar{\lambda}^{2}\right)$ has the

\footnotetext{
${ }^{2}$ The cusp anomalous dimension is known up to two-loops, see 37.
} 
Landau pole. This feature can be treated in terms of IR renormalon ambiguities (see next Section), and is often considered as a signal of nonperturbative physics. In the present paper, we will consistently separate the sources of nonperturbative effects which can be attributed to uncertainties of resummation of the perturbative series from the "true" nonperturbative phenomena. An important example of the latter is provided by instanton induced effects within the instanton model of QCD vacuum, which is considered in the Section 6 .

\section{IR renormalon induced effects}

As it was pointed out at the end of the previous Section, the perturbative evolution equation (4.17) possesses an unphysical singularity at the point $\lambda^{2}=1$. Therefore, it is instructive to study the consequences of this feature. It is known that the presence of the Landau pole in the one-loop expression for the coupling constant leads to the IR renormalons [42] resulting in power suppressed corrections. In the present situation one can expect the corrections proportional to the powers of both scales: $\mu^{2}$ and $\lambda^{2}$. We will treat here the power $\mu^{2}$-terms to be strongly suppressed in large- $q^{2}$ regime, and focus on the power $\lambda^{2}$ corrections. To find them, let us consider the perturbative function $\Delta_{1}\left(\varepsilon, 0, \bar{\mu}^{2} / \lambda^{2}\right)$ in the Eq. (4.9). The insertion of the fermion bubble 1-chain to the one-loop order expression (4.4) is equivalent to replacement of the frozen coupling constant $g^{2}$ by the running one $g^{2} \rightarrow g^{2}\left(k^{2}\right)=4 \pi \alpha_{s}\left(k^{2}\right)$ 48 (for convenience, we work here in Euclidean space.):

$$
\widetilde{\Delta}_{1}\left(\varepsilon, 0, \bar{\mu}^{2} / \lambda^{2}\right)=-4 \pi \bar{\mu}^{2 \varepsilon} \int \frac{d^{n} k}{(2 \pi)^{n}} \alpha_{s}\left(k^{2}\right) \frac{\mathrm{e}^{i k z} \delta\left(z^{2}\right)}{k^{2}\left(k^{2}+\lambda^{2}\right)} .
$$

By using the integral representation for the one-loop running coupling $\alpha_{s}\left(k^{2}\right)=\int_{0}^{\infty} d \sigma\left(1 / k^{2}\right)^{\sigma b}$, $b=\beta_{0} / 4 \pi$, we find:

$$
\widetilde{\Delta}_{1}\left(\varepsilon, 0, \bar{\mu}^{2} / \lambda^{2}\right)=-\frac{1}{\beta_{0}(1-\varepsilon)}\left(4 \pi \frac{\bar{\mu}^{2}}{\lambda^{2}}\right)^{\varepsilon} \int_{0}^{\infty} d x \frac{\Gamma(1-x-\varepsilon) \Gamma(1+x+\varepsilon)}{(x+\varepsilon) \Gamma(1-\varepsilon)}\left(\frac{1}{\lambda^{2}}\right)^{x} .
$$

To define properly the integral in r. h. s. of Eq.(5.2), one needs to specify a prescription to go around the poles, which are at the points $\bar{x}_{n}=n, n \in \mathbb{N}$. Thus, the result of integration depends on this prescription giving an ambiguity proportional to $\left(1 / \lambda^{2}\right)^{n}$ for each pole. Then, the IR renormalons produce the power corrections to the one-loop perturbative

result, which we assume to exponentiate with the latter 48. Extracting from (5.2) the UV singular part in vicinity of the origin $x=0$, we divide the integration interval $[0, \infty]$ in two parts: $[0, \delta]$ and $[\delta, \infty]$, where $\delta<1$. This procedure allows us to evaluate separately the ultraviolet and the renormalon-induced pieces. For the ultraviolet piece, we apply the expansion of the integrand in $\Delta_{1}$ in powers of small $x$ and replace the ratio of $\Gamma$-functions by $\exp \left(-\gamma_{E} \varepsilon\right)$ :

$$
\widetilde{\Delta}_{1}^{U V}\left(\varepsilon, 0, \bar{\mu}^{2} / \lambda^{2}\right)=-\frac{1}{\beta_{0}(1-\varepsilon)} \sum_{k, n=0}(-)^{n} \frac{\left(\ln 4 \pi-\gamma_{E}+\ln \frac{\bar{\mu}^{2}}{\lambda^{2}}\right)^{k}}{k ! \varepsilon^{n-k+1}} \int_{0}^{\delta} d x x^{n}\left(\frac{1}{\lambda^{2}}\right)^{x},
$$


which after subtraction of the poles in the $\overline{M S}$-scheme becomes:

$$
\widetilde{\Delta}_{1}^{U V}\left(0, \mu^{2} / \lambda^{2}\right)=\frac{1}{\beta_{0}(1-\varepsilon)} \sum_{n=1}\left(\ln \frac{\mu^{2}}{\lambda^{2}}\right)^{n} \frac{(-)^{n}}{n !} \int_{0}^{\delta} d x x^{n-1}\left(\frac{1}{\lambda^{2}}\right)^{x} .
$$

In analogy with results of [49], this expression may be rewritten in a closed form as

$$
\widetilde{\Delta}_{1}^{U V}\left(0, \mu^{2} / \lambda^{2}\right)=\frac{1}{\beta_{0}(1-\varepsilon)} \int_{0}^{\delta} \frac{d x}{x}\left[\mathrm{e}^{-x \ln \mu^{2}}-\mathrm{e}^{-x \ln \lambda^{2}}\right] .
$$

Then, using the relation

$$
\frac{\partial W^{(1)}\left(q^{2}\right)}{\partial \ln q^{2}}=2 C_{F}(1-\varepsilon) \widetilde{\Delta}_{1}^{U V}\left(0, \mu^{2} / \lambda^{2}\right)
$$

one finds

$$
\left(\mu^{2} \frac{\partial}{\partial \mu^{2}}+\beta(g) \frac{\partial}{\partial g}\right) \frac{\partial W^{(1)}\left(q^{2}\right)}{\partial \ln q^{2}}=-\frac{1}{2} \Gamma_{c u s p}^{(1)}\left(\alpha_{s}\left(\mu^{2}\right)\right)\left(1-\exp \left[-\delta \frac{4 \pi}{\beta_{0} \alpha_{s}\left(\mu^{2}\right)}\right]\right) .
$$

The exponent in the last equation yields the power suppressed terms $\left(1 / q^{2}\right)^{\delta}$ in large- $q^{2}$ regime. In LLA the Eq. (5.6) is reduced to:

$$
\frac{\partial W^{(1)}\left(q^{2}\right)}{\partial \ln q^{2}}=-\frac{2 C_{F}}{\beta_{0}}\left(\ln \frac{\ln \mu^{2}}{\ln \lambda^{2}}\right) .
$$

The last expression obviously satisfies the perturbative evolution equation (4.17).

The remaining integral in Eq. (5.2) over the interval $[\delta, \infty]$ is evaluated at $\varepsilon=0$ since there are no UV singularities. The resulting expression does not depend on the normalization point $\mu$, and thus it is determined by the IR region including nonperturbative effects. It contains the renormalon ambiguities due to different prescriptions in going around the poles $\bar{x}_{n}$ in the Borel plane which yields the power corrections to the quark form factor.

After the substitution $\mu^{2}=q^{2}$ and integration, we find in LLA (for comparison, see Eq. (4.17)):

$$
F_{q}^{r e n}\left(q^{2}\right)=\exp \left[-\frac{2 C_{F}}{\beta_{0}} \ln q^{2}\left(\ln \ln q^{2}-1\right)-\ln q^{2} \Phi_{r e n}\left(\lambda^{2}\right)\right] F^{r e n}\left(q_{0}^{2}\right),
$$

where the function $\Phi_{\text {ren }}\left(\lambda^{2}\right)=\sum_{k=0} \phi_{k}\left(1 / \lambda^{2}\right)^{k}$ accumulates the effects of the IR renormalons. The coefficients $\phi_{k}$ cannot be calculated in perturbation theory and are often treated as "the minimal set" of nonperturbative parameters. It is worth noting that the logarithmic $q^{2}$-dependence of the renormalon corrections in the large- $q^{2}$ regime is factorized, and thus the Eq. (5.9) corresponds to the structure of nonperturbative contributions found in the one-loop evolution equation (4.17). On the other hand, it can be shown that careful account of partially resumed perturbative series yields, sometimes, cancellation of the leading power corrections associated with the leading renormalon contributions [48]. As the corresponding nonperturbative terms are calculated independently (e.g., by means 
of the ILM) their direct relation to the IR renormalon ambiguities should be questioned. In our point of view, it allows us to separate the true nonperturbative (e.g., instanton induced, but not only) effects from that ones related to ambiguities of the resumed perturbative series.

The latter conclusion can be illustrated by considering the consequences of an analytization (AN) of the strong coupling constant [43] in the perturbative evolution equation. In this approach, the one-loop strong coupling constant $\alpha_{s}\left(\mu^{2}\right)$ is replaced by the expression which is analytical at $\mu^{2}=1$ (i.e., at $\Lambda_{Q C D}^{2}$ in dimensional variables):

$$
\alpha_{s}^{A N}\left(\mu^{2}\right)=\frac{4 \pi}{\beta_{0}}\left(\frac{1}{\ln \mu^{2}}+\frac{1}{1-\mu^{2}}\right) .
$$

The direct substitution of (5.10) into the evolution equation (3.14) yields (for brevity, we assume $\left.q_{0}^{2}=\lambda^{2}\right)$ :

$$
\begin{aligned}
& -\left(\frac{\beta_{0}}{2 C_{F}}\right) \ln F_{q}^{A N}\left(q^{2}\right)=\ln q^{2}\left(\ln \frac{\ln q^{2}}{\ln \lambda^{2}}-1\right)-\frac{3}{2} \ln \frac{\ln q^{2}}{\ln \lambda^{2}}+ \\
& +\left(\ln q^{2}+\frac{3}{2}\right)\left(\ln \frac{q^{2}\left(\lambda^{2}-1\right)}{\left(q^{2}-1\right) \lambda^{2}}\right)-\frac{1}{2}\left(\ln ^{2} q^{2}-\ln ^{2} \lambda^{2}\right)-\operatorname{Li}_{2}\left(1-q^{2}\right)+\operatorname{Li}_{2}\left(1-\lambda^{2}\right)
\end{aligned}
$$

The functions $\mathrm{Li}_{2}$ in the resulting expression accumulate the power corrections of $q^{2}$ and IR scale $\lambda^{2}$, but does not exhibit a singularity at $\lambda^{2}=1$. Therefore, it gives no room for IR renormalons ambiguities, at least in the considered approximation. Nevertheless, the power corrections of nonperturbative origin do contribute to the large- $q^{2}$ behaviour. Note, that the consequences of the analytization of the strong coupling constant in the IR region have been studied also in 50 .

Another possible way to avoid the Landau pole at the integration path was developed within the dimensional regularization (DR) scheme [24]. In this case, the running coupling reads

$$
\alpha_{s}^{D R}\left(\varepsilon ; \mu^{2}\right)=\frac{4 \pi \varepsilon}{\beta_{0}\left[\left(q^{2}\right)^{\varepsilon}-1\right]},
$$

and for complex $\varepsilon$, Re $\varepsilon<0$, it has the Landau pole at the complex value of $\mu^{2}$, thus this singularity moves out of the integration contour. In the limit $\varepsilon \rightarrow 0$, the form factor reads 24] (for comparison, see Eq. 4.17):

$$
\begin{gathered}
F_{q}^{D R}\left(q^{2}\right)= \\
\exp \left[-\frac{2 C_{F}}{\beta_{0}}\left(\frac{\zeta(2)}{\varepsilon}+\ln \varepsilon\left(\ln q^{2}-\frac{3}{2}\right)+\ln q^{2}\left(\ln \ln q^{2}-1\right)-\frac{3}{2} \ln \ln q^{2}\right)+O(\varepsilon, \varepsilon \ln \varepsilon)\right] .
\end{gathered}
$$

This expression also leaves no room for any renormalon effects. At the same time, the nonperturbative contributions still take place since they enter into the "integration constant" $W_{n p}, 3.11$, which is not directly related to the analytical properties of the coupling constant. 


\section{Instanton induced contribution to the Wilson integral}

In this Section we consider explicitly the nonperturbative effects within the instanton model of QCD vacuum. The instanton effects in the high energy QCD processes has been actively studied since the seventies [51, 52]. Recently, the investigation of these effects was renewed with promising perspectives [26, 46, 47, 53, 54, 55, 56, 57, 58, 59, 60]. The Wilson integral formalism is considered as a useful and convenient tool in the instanton applications mainly due to significant simplification in the path integral calculations if an explicit form of the gauge field is known. Another important feature of this approach is the possibility to make a correct analytical continuation of the results obtained in the Euclidean space (where the instantons are only determined) to the physical Minkowski space-time where the scattering processes actually take place. Namely, one maps the scattering angle, $\chi$, to the Euclidean space angle, $\gamma$, by analytical continuation [61]

$$
\chi \rightarrow i \gamma
$$

and performs the inverse transformation to the Minkowski space-time in the final expressions in order to restore the $q^{2}$-dependence.

Let us consider the instanton contribution to the function $W_{n p}\left(q^{2}\right)$ from Eq.(3.13). The instanton field has general form

$$
\hat{A}_{\mu}(x ; \rho)=A_{\mu}^{a}(x ; \rho) \frac{\sigma^{a}}{2}=\frac{1}{g} \mathbb{R}^{a b} \sigma^{a} \eta^{ \pm}{ }_{\mu \nu}\left(x-z_{0}\right)_{\nu} \varphi\left(x-z_{0} ; \rho\right),
$$

where $\varphi_{I}(x)$ is the gauge dependent profile function, $\mathbb{R}^{a b}$ is the color orientation matrix, $\sigma^{a}$ 's are the Pauli matrices, $\eta_{\mu \nu}^{ \pm a}=\varepsilon_{4 a \mu \nu} \mp(1 / 2) \varepsilon_{a b c} \varepsilon_{b c \mu \nu}$ are 't Hooft symbols, and $( \pm)$ corresponds to the instanton or antiinstanton solution ${ }^{3}$.

The averaging of the Wilson operator over the nonperturbative vacuum is performed by the integration over the coordinate of the instanton center $z_{0}$, the color orientation and the instanton size $\rho$. The measure for the averaging over the instanton ensemble reads $d I=d \mathbb{R} d^{4} z_{0} d n(\rho)$, where $d \mathbb{R}$ refers to the averaging over color orientation, and $d n(\rho)$ depends on the choice of the instanton size distribution. Taking into account (6.2), we write the Wilson integral (3.2) with contour (3.3) in the single instanton approximation in the form:

$$
\begin{aligned}
w_{I}(\chi) & =\frac{1}{N_{c}} \operatorname{Tr}\left\langle 0\left|\exp \left[i \sigma^{a}\left(\hat{n}_{1}^{a} \alpha\left(v_{1}, z_{0}\right)+\hat{n}_{2}^{a} \alpha\left(v_{2}, z_{0}\right)\right)\right]\right| 0\right\rangle= \\
& =\frac{1}{N_{c}} \operatorname{Tr}\langle 0| \cos \left(\alpha\left(v_{1}, z_{0}\right)\right) \cos \left(\alpha\left(v_{2}, z_{0}\right)\right)(\mathbb{I} \times \mathbb{I})- \\
& -\frac{1}{3}\left(\hat{n}_{1} \cdot \hat{n}_{2}\right) \sin \left(\alpha\left(v_{1}, z_{0}\right)\right) \sin \left(\alpha\left(v_{2}, z_{0}\right)\right)\left(\sigma^{a} \times \sigma^{a}\right)|0\rangle,
\end{aligned}
$$

where $(i=1,2)$

$$
\hat{n}_{i}^{a}=\frac{(-1)^{i}}{s\left(v_{i}, z_{0}\right)} \eta_{\mu \nu}^{ \pm a} v_{i}^{\mu} z_{0}^{\nu}
$$

\footnotetext{
${ }^{3}$ Below we always consider only topologically neutral instanton (I) and antiinstanton (A) configurations, but since there are no differences in contributions of I and A in the processes considered both solutions will be called as instanton.
} 


$$
\alpha\left(v_{i}, z_{0}\right)=s\left(v_{i}, z_{0}\right) \int_{0}^{\infty} d \lambda \varphi\left[\left(v_{i} \lambda+(-1)^{i} z_{0}\right)^{2} ; \rho_{c}\right]
$$

and

$$
s^{2}\left(v_{i}, z_{0}\right)=z_{0}^{2}-\left(v_{i} z_{0}\right)^{2}
$$

where $\left(v_{1} v_{2}\right)=\cosh \chi$ in Minkowski geometry. We omit the path ordering operator $\mathcal{P}$ in (6.3) because the instanton field (6.2) is a hedgehog in color space, and so it locks the color orientation by space coordinates. Let us note that due to nonperturbative factor $g^{-1}$ in the instanton field (6.2) the Wilson integral (6.3) is independent on the coupling constant.

At this stage we need to average over all possible ways of embedding $S U_{c}(2)$ into $S U_{c}(3)$ by using the correspondence relations 62

$$
\begin{aligned}
\left\langle(\mathbb{I} \times \mathbb{I})_{S U(2)}\right\rangle_{S U(3)} & =\frac{4}{9}\left[(\mathbb{I} \times \mathbb{I})_{S U(3)}+\frac{3}{32}\left(\lambda^{A} \times \lambda^{A}\right)_{S U(3)}\right], \\
\left\langle\left(\sigma^{a} \times \sigma^{a}\right)_{S U(2)}\right\rangle_{S U(3)} & =\frac{3}{8}\left(\lambda^{A} \times \lambda^{A}\right)_{S U(3)},
\end{aligned}
$$

where $\lambda^{A}$ are generators of $S U(3)$ algebra $(A=1,2, \ldots, 8)$. Then we get

$$
\begin{aligned}
w_{I}(\chi) & =\frac{1}{3} \operatorname{Tr}\langle 0| \frac{4}{9} \cos \left(\alpha\left(v_{1}, z_{0}\right)\right) \cos \left(\alpha\left(v_{2}, z_{0}\right)\right)(\mathbb{I} \times \mathbb{I})+ \\
& +\frac{1}{8}\left[\frac{1}{3} \cos \left(\alpha\left(v_{1}, z_{0}\right)\right) \cos \left(\alpha\left(v_{2}, z_{0}\right)\right)-\sin \left(\alpha\left(v_{1}, z_{0}\right)\right) \sin \left(\alpha\left(v_{2}, z_{0}\right)\right) \hat{n}_{1}^{a} \hat{n}_{2}^{a}\right]\left(\lambda^{A} \times \lambda^{A}\right)|0\rangle .
\end{aligned}
$$

The resulting gauge invariant contribution to the Wilson loop of the single instanton taken in all orders in gauge field becomes 46]

$$
\begin{aligned}
& w_{I}(\chi)=\frac{2}{3} \int d n(\rho)\left[w_{c}^{I}(\chi)+w_{s}^{I}(\chi)-w_{c}^{I}(0)-w_{s}^{I}(0)\right], \\
& w_{c}^{I}(\chi)=\int d^{4} z_{0} \cos \alpha\left(v_{1}, z_{0}\right) \cos \alpha\left(v_{2}, z_{0}\right), \\
& w_{s}^{I}(\chi)=-\int d^{4} z_{0}\left(\hat{n}_{1}^{a} \hat{n}_{2}^{a}\right) \sin \alpha\left(v_{1}, z_{0}\right) \sin \alpha\left(v_{2}, z_{0}\right),
\end{aligned}
$$

where the normalized color correlation factor is

$$
\hat{n}_{1}^{a} \hat{n}_{2}^{a}=-\frac{\eta_{\mu \nu}^{a} v_{1}^{\mu} z_{0}^{\nu} \eta_{\rho \sigma}^{a} v_{2}^{\rho} z_{0}^{\sigma}}{s\left(v_{1}, z_{0}\right) s\left(v_{2}, z_{0}\right)} .
$$

The Eq. (6.9) takes into account the subtraction of the infinite self-energy parts of the quark form factor. Thus, to calculate the instanton contribution one need to consider only the vertex corrections.

\section{Exponentiation of the instanton contributions in the dilute regime}

On the basis of the exponentiation theorem [40] for the non-Abelian path-ordered exponentials it is well known that perturbative corrections to the Sudakov form factor are 
exponentiated to high orders in the QCD coupling constant. The theorem states that the contour average $W_{P}(C)$ can be expressed as

$$
W_{P}(C)=\exp \left[\sum_{n=1}^{\infty}\left(\frac{\alpha_{s}}{\pi}\right)^{n} \sum_{W \in W(n)} C_{n}(W) F_{n}(W)\right]
$$

where summation in the exponential is over all diagrams $W$ of the set $W(n)$ of the twoparticle irreducible contour averages of $n$th order of the perturbative expansion. The coefficients $C_{n}(W) \propto C_{F} N_{c}^{n-1}$ are the "maximally non-Abelian" parts of the color factor corresponding to the contribution coming from a diagram $W$ to the total expression (7.1) in the contour gauge ${ }^{4}$, and the factor $F_{n}(W)$ is the contour integral presented in the expression for $W$. This means that the essential diagrams are only those, which do not contain the lower-order contributions as subgraphs and, as a result, the higher-order terms are non-Abelian.

Let us now demonstrate how the single instanton contribution is exponentiated in the small instanton density parameter, treating the instanton vacuum as a dilute medium 64. The gauge field is taken to be the sum of individual instanton fields in the singular gauge, with their centers at the points $z_{j}$ 's. In this gauge, the instanton fields fall off rapidly at infinity, so the instantons may be considered individually in their effect on the Wilson loop. Moreover, the contribution of infinitely distant parts of the contour may be neglected and only those instantons will influence the loop integral, which occupy regions of space-time intersecting with the quark trajectories. Since the parameterization of the loop integral along rays of the angle plays the role of the proper time, a time-ordered series of instantons arises and has an effect on the Wilson loop. Thus, the contribution of $n$ instantons to the loop integral $W_{I}(\chi)$ can be written in the dilute approximation as

$$
W_{I}^{(n)}(\chi)=\operatorname{Tr}\left(U^{1} U^{2} \ldots U^{n} U^{n \dagger} \ldots U^{2 \dagger} U^{1 \dagger}\right)
$$

where the ordered line integrals $U_{i}$ 's

$$
U^{j}(\chi)=T\left\{\exp \left(i g \int_{0}^{\infty} d \sigma v_{1}^{\mu} A_{\mu}\left(v_{1} \sigma-z_{j}\right)\right) \exp \left(i g \int_{-\infty}^{0} d \tau v_{2}^{\mu} A_{\mu}\left(v_{2} \tau-z_{j}\right)\right)\right\}
$$

are associated with individual instantons with the positions $z_{j}$ 's. Because of the wide separation of the instantons in the dilute phase and rapid fall off of fields in the singular gauge, the upper and lower limits of the line integrals are extended to infinity. The line integrals $U^{i \dagger}$ 's take into account the infinitely distant part of the contour that goes from $+\infty$ back to $-\infty$ and in the singular gauge they are $U^{i \dagger}=1$. For $U^{j}\left(U^{j \dagger}\right)$, the integral is taken over the increasing (decreasing) time piece of the loop.

Then, the expression is further simplified by averaging over the gauge orientations of instantons. The averaging is reduced to substitution of $U^{j}$ by $g_{j} U^{j} g_{j}^{-1}$, where $g_{j}$ is

\footnotetext{
${ }^{4}$ The contour gauge is defined as a gauge where the condition $P \exp \left(i g \int_{x_{0}}^{x} d z_{\mu} \hat{A}_{\mu}(z)\right)=1$ is fulfilled 63.
} 
an element of colour group, and independent integration of each $g_{j}$ over the properly normalized group measure is performed. Under this averaging one gets

$$
U^{n} U^{n \dagger} \rightarrow w_{I}^{(n)}(\chi)=\frac{1}{N_{c}} \operatorname{Tr}\left(U^{n} U^{n \dagger}\right)
$$

which is just the single instanton contribution as it is given by Eqs. (6.9). If the averaging is done in the inverse order, from $n$ down to 1 , the entire loop integral becomes the product of traces

$$
W_{I}^{(n)}(\chi) \rightarrow \lim _{n \rightarrow \infty} \prod_{j=1}^{n} w_{I}^{(j)}(\chi) .
$$

Since the individual instantons are considered to be decoupled in the dilute medium, the total multiple instanton contribution to the vacuum average of the Wilson operator simply exponentiates the all-order single instanton term $w_{I}(\chi)$ in (6.9), and one has

$$
W_{I}(\chi)=\lim _{n \rightarrow \infty}\left\{1+\frac{1}{n} w_{I}(\chi)\right\}^{n}=\exp \left[w_{I}(\chi)\right]
$$

Thus, we prove that in the dilute regime the full instanton contribution to the quark form factor is given by the exponent of the all-order single instanton result (see Fig. 1e). The exponentiation arises due to taking into account the multi-instanton configurations. As it is well known, in QED there occurs the exponentiation of the one-loop result due to Abelian character of the theory. In the instanton case, the analogous result arises since instantons belong to the $S U(2)$ subgroup of the $S U(3)$ color group and the path-ordered exponents coincide with the ordinary ones.

The following comments are in order. First, the nonperturbative exponentiated expressions are strictly correct only as long as the instanton density $n(\rho)$ is small. Second, it is supposed that $U\left(z_{0}\right)$ is evaluated using the singular gauge form of $A_{\mu}^{\text {inst }}$. On the other hand, $\operatorname{Tr}\left(U U^{\dagger}\right)$ is identically the ordered loop integral for a single instanton and is gauge invariant. It is therefore legal to use the nonsingular gauge of $A_{\mu}^{\text {inst }}$ in evaluating the trace (a more handing gauge for computation).

\section{Large $-q^{2}$ behaviour of the instanton contribution in the weak field ap- proximation}

Let us first consider the weak-field approximation to the instanton contribution to the quark form factor. In this limit the leading instanton induced term in Eq. (6.9) is given by general expression (4.4) (Fig. 20) with the corresponding dimensionally regularized instanton correlator

$$
\mathcal{D}_{\mu \nu}^{I}(z)=\lambda^{n-4} \int d n(\rho) \int \frac{d^{n} k}{(2 \pi)^{n}} \tilde{A}_{\mu}^{a}(k ; \rho) \tilde{A}_{\nu}^{a}(-k ; \rho) \mathrm{e}^{-i k z} .
$$

By using the Fourier transform of the instanton field embedded into the $S U(3)$ color group

$$
\tilde{A}_{\mu}^{a}(k ; \rho)=-\frac{2 i}{g} \mathbb{R}^{a \alpha} \eta_{\mu \sigma}^{ \pm \alpha} k_{\sigma} \tilde{\varphi}^{\prime}\left(k^{2} ; \rho\right)
$$


where $\tilde{\varphi}\left(k^{2} ; \rho\right)$ is the Fourier transform of the instanton profile function $\varphi\left(z^{2} ; \rho\right)$ and $\tilde{\varphi}^{\prime}\left(k^{2} ; \rho\right)$ is its derivative with respect to $k^{2}$, and the properties of the color rotation matrices and 't Hooft symbols

$$
\mathbb{R}^{a \beta} \mathbb{R}^{a \gamma}=\delta^{\beta \gamma}, \eta_{\mu \rho}^{\alpha} \eta_{\nu \lambda}^{\alpha}=g_{\mu \nu} g_{\rho \lambda}-g_{\mu \lambda} g_{\nu \rho}+\varepsilon_{\mu \nu \rho \lambda}
$$

one can express the instanton correlator (8.1) in the form similar to Eq. (4.8)

$$
\mathcal{D}_{\mu \nu}^{I}(z)=\frac{1}{g^{2}}\left(g_{\mu \nu} \partial^{2}-\partial_{\mu} \partial_{\nu}\right) \Delta_{I}\left(z^{2}\right)
$$

where

$$
\Delta_{I}\left(z^{2}\right)=-\lambda^{n-4} \int d n(\rho) \frac{d^{n} k}{(2 \pi)^{n}} \mathrm{e}^{-i k z}\left[2 \tilde{\varphi}^{\prime}\left(k^{2} ; \rho\right)\right]^{2} .
$$

Then, applying the same method as described in perturbative case, one gets the LO instanton contribution in the form

$$
w_{I}^{(L O)}(\chi)=\frac{1}{N_{c}} h(\chi) \int d n(\rho) \Delta_{I}\left(0, \rho^{2} \lambda^{2}\right),
$$

where we use the same IR cutoff $\lambda^{2}$, while the UV divergences do not appear at all due to the finite instanton size. In the singular gauge with the profile function given by

$$
\varphi_{I}(x)=\frac{\rho^{2}}{x^{2}\left(x^{2}+\rho^{2}\right)},
$$

one gets:

$$
\Delta_{I}\left(0, \rho^{2} \lambda^{2}\right)=\frac{\pi^{2} \rho^{4}}{4}\left[\ln \left(\rho^{2} \lambda^{2}\right) \Phi_{0}\left(\rho^{2} \lambda^{2}\right)+\Phi_{1}\left(\rho^{2} \lambda^{2}\right)\right]
$$

where

$$
\Phi_{0}\left(\rho^{2} \lambda^{2}\right)=\frac{1}{\rho^{4} \lambda^{4}} \int_{0}^{1} \frac{d z}{z(1-z)}\left[1+\mathrm{e}^{\rho^{2} \lambda^{2}}-2 \mathrm{e}^{z \cdot \rho^{2} \lambda^{2}}\right] \quad, \quad \lim _{\lambda^{2} \rightarrow 0} \Phi_{0}\left(\rho^{2} \lambda^{2}\right)=1,
$$

and

$$
\Phi_{1}\left(\rho^{2} \lambda^{2}\right)=\sum_{n=1}^{\infty} \int_{0}^{1} d x d y d z \frac{\left[-\rho^{2} \lambda^{2}(x z+y(1-z))\right]^{n}}{n ! n} \mathrm{e}^{\rho^{2} \lambda^{2}[x z+y(1-z)]}, \quad \lim _{\lambda^{2} \rightarrow 0} \Phi_{1}\left(\rho^{2} \lambda^{2}\right)=0
$$

are the IR-finite expressions.

At high energy the instanton contribution is reduced to the form:

$$
\frac{\partial \ln W_{I}\left(q^{2}\right)}{\partial \ln q^{2}}=\frac{\pi^{2}}{4 N_{c}} \int d n(\rho) \rho^{4}\left[\ln \left(\rho^{2} \lambda^{2}\right) \Phi_{0}\left(\rho^{2} \lambda^{2}\right)+\Phi_{1}\left(\rho^{2} \lambda^{2}\right)\right] \equiv-B_{I}\left(\lambda^{2}\right) .
$$

Here we used the exponentiation of the single-instanton result in a dilute instanton ensemble (see [46] and the previous Section) and took only the LO term of the weak-field expansion (4.4): $W^{(1)}=w_{I}+$ (higher order terms).

In order to estimate the magnitude of the instanton effect we take the instanton size distribution found by 't Hooft [65] and multiply it by the exponential suppressing factor 
(which was suggested in [67] in the framework of constrained instanton model and assumed in [68] in order to describe the lattice data [10]) :

$$
d n(\rho)=\frac{d \rho}{\rho^{5}} C_{N_{c}}\left[\frac{2 \pi}{\alpha_{s}\left(\mu_{r}\right)}\right]^{2 N_{c}} \exp \left[-\frac{2 \pi}{\alpha_{s}\left(\mu_{r}\right)}\right]\left(\rho \mu_{r}\right)^{\beta} \exp \left(-2 \pi \sigma \rho^{2}\right),
$$

where the constant $C_{N_{c}}=4.6 / \pi^{2} \exp \left(-1.679 N_{c}\right) /\left[\left(N_{c}-1\right) !\left(N_{c}-2\right) !\right] \approx 0.0015, \sigma \approx$ $(0.44 \mathrm{GeV})^{2}$ is the string tension, $\beta=\beta_{0}+O\left(\alpha_{s}\left(\mu_{r}\right)\right)$, and $\mu_{r}$ is the normalization point [69.

Given the distribution (8.11) the main parameters of the instanton liquid model, the instanton density $n_{c}$ and the mean instanton size $\rho_{c}$, will read:

$$
\begin{gathered}
n_{c}=\int_{0}^{\infty} d n(\rho)=\frac{C_{N_{c}} \Gamma(\beta / 2-2)}{2}\left[\frac{2 \pi}{\alpha_{S}\left(\bar{\rho}^{-1}\right)}\right]^{2 N_{c}}\left[\frac{\Lambda_{Q C D}}{\sqrt{2 \pi \sigma}}\right]^{\beta}(2 \pi \sigma)^{2}, \\
\rho_{c}=\frac{\int_{0}^{\infty} \rho d n(\rho)}{\int_{0}^{\infty} d n(\rho)}=\frac{\Gamma(\beta / 2-3 / 2)}{\Gamma(\beta / 2-2)} \frac{1}{\sqrt{2 \pi \sigma}} .
\end{gathered}
$$

In Eq. (8.13) we choose, for convenience, the normalization scale $\mu_{r}$ of order of the instanton inverse mean size $\rho_{c}^{-1}$, taking into account that the distribution function (8.11) is the RGinvariant quantity up to $O\left(\alpha_{s}^{2}\right)$ terms [69]. Note, that these quantities correspond to the mean size $\rho_{c}$ and density $n_{c}$ of instantons used in the model [6], where the size distribution (8.11) is approximated by the delta-function:

$$
d n(\rho)=n_{c} \delta\left(\rho-\rho_{c}\right) d \rho
$$

Thus, we find the leading instanton contribution (8.10) in the form:

$$
B_{I}\left(\lambda^{2}\right)=\frac{K f}{2 N_{c}} \ln \frac{2 \pi \sigma}{\lambda^{2}}\left[1+O\left(\frac{\lambda^{2}}{2 \pi \sigma}\right)\right]
$$

where

$$
K=\frac{\Gamma\left(\beta_{0} / 2\right)\left[\Gamma\left(\beta_{0} / 2-2\right)\right]^{3}}{2\left[\Gamma\left(\beta_{0} / 2-3 / 2\right)\right]^{4}} \approx 0.74
$$

and we used the one loop expression for the running coupling constant

$$
\alpha_{s}\left(\rho_{c}^{-1}\right)=-\frac{2 \pi}{\beta_{0} \ln \rho_{c} \Lambda} .
$$

In 8.15$)$ we introduces the packing fraction parameter

$$
f=\pi^{2} n_{c} \rho_{c}^{4}
$$

that characterizes diluteness of the instanton liquid. Within the conventional picture its value is estimated to be

$$
f \approx 0.12
$$

if one takes the model parameters as (see [8]):

$$
n_{c} \approx 1 \mathrm{fm}^{-4}, \quad \rho_{c} \approx 1 / 3 \mathrm{fm}
$$


Therefore, from Eqs. (8.10) and (5.9), we find the expression for the quark form factor at large- $q^{2}$ with the one-loop perturbative contribution and the nonperturbative contribution (the function $W_{n p}$ in Eq. (4.17)) found in the instanton model:

$$
F_{q}\left(q^{2}\right)=\exp \left[-\frac{2 C_{F}}{\beta_{0}} \ln q^{2} \ln \ln q^{2}-\ln q^{2}\left(B_{I}-\frac{2 C_{F}}{\beta_{0}}\right)+O\left(\ln \ln q^{2}\right)\right] F_{0}\left(q_{0}^{2} ; \lambda^{2}\right) .
$$

Numerically the coefficient $B_{I}$ is small factor as compared to the perturbative term

$$
B_{I} \approx 0.03 \ll \frac{2 C_{F}}{\beta_{0}} \approx 0.24,
$$

where to get this estimate we take the IR cutoff parameter $\lambda$ of order of $\Lambda_{Q C D} \approx 350$ $\mathrm{MeV}$. It is clear, that while the asymptotic "double-logarithmic" behaviour is controlled by the perturbative cusp anomalous dimension, the leading instanton correction results in a finite renormalization of the subleading perturbative term (Fig.3). Note, that the instanton correction has the opposite sign compared to the perturbative logarithmic term.

It is also important to note that the results obtained are quite sensitive to the way one makes the integration over instanton sizes finite. For example, if one used the sharp cutoff then the instanton would produce strongly suppressed power corrections like $\propto(1 / q)^{\beta_{0}}$. However, we think that the distribution function (8.11) should be considered as more realistic, since it reflects more properly the structure of the instanton ensemble modeling the QCD vacuum. Indeed, this shape of distribution was recently advocated in [68, 67] and supported by the lattice calculations [10] (for comparison, see, however, [70, 71]).

\section{All-order calculations of the Wilson loop for Gaussian profile}

The weak field limit used in the previous Section may deviate from the exact result. Here, we are going to test its accuracy considering the properties of the single instanton contribution to the Wilson loop, $w_{I}(\chi)$, defined in Eq. (6.9), which contains contributions from the gauge field taken in all orders.

We have to note that in realistic instanton vacuum model there are two essential effects: stabilization of the instanton density with respect to unbounded expansion of instantons in size (see Eqs. (8.11) and (8.14), and screening of instantons by surrounding background fields. To take into account these features we approximate first the narrow instanton size distribution by the $\delta$ - function as in Eq. (8.14) and assume that the integration over the instanton size is already performed. As it was discussed above, the screening effect modifies the instanton shape at large distances leading to the constrained instantons [67]. To take into account this screening and to have also simpler analytical form for $w_{I}(\chi)$, we use in this Section the Gaussian Anzatz for the instanton profile function

$$
\varphi_{G}\left(x^{2}\right)=\frac{1}{\rho_{c}^{2}} e^{-x^{2} / \rho_{c}^{2}}
$$

The parameters in this expression are fixed from the requirement to reproduce the vacuum average value of the $\left\langle A_{\mu}^{a}(0) A_{\nu}^{a}(0)\right\rangle$ which is finite for the instanton field in the singular 
gauge and equals ${ }^{5}$ :

$$
\left\langle g^{2} A_{\mu}^{a}(0) A_{\mu}^{a}(0)\right\rangle=12 \pi^{2} \rho_{c}^{2} n_{c} .
$$

Below we equal the parameter $\rho_{c}$ to unity and restore the dependence on it at the end of calculations.

The phases (6.5) corresponding to the profile (9.1) are calculated as

$$
\alpha\left(v_{1,2}, z_{0}\right)=s\left(v_{1,2}, z_{0}\right) \frac{\sqrt{\pi}}{2} e^{-s^{2}\left(v_{1,2}, z_{0}\right)} \operatorname{erfc}\left[\mp\left(v_{1,2} z_{0}\right)\right],
$$

where with definitions $(3.3)$ one has ${ }^{6}$

$$
\begin{aligned}
\left(v_{1} z_{0}\right) & =z_{4}, \quad\left(v_{2} z_{0}\right)=z_{4} \cosh \chi+i z_{3} \sinh \chi \\
s^{2}\left(v_{1}, z_{0}\right) & =z_{3}^{2}+z_{\perp}^{2}, \quad s^{2}\left(v_{2}, z_{0}\right)=\left(z_{3} \cosh \chi-i z_{4} \sinh \chi\right)^{2}+z_{\perp}^{2} .
\end{aligned}
$$

We take the reference frame where the scattering point is in the origin and define the scattering vectors of the quark as

$$
\begin{gathered}
v_{1}=\left(1,0,0_{\perp}\right), \quad v_{2}=\left(\cosh \chi, i \sinh \chi, 0_{\perp}\right), \\
v_{1}^{2}=v_{2}^{2}=1, \quad\left(v_{1} v_{2}\right)=\cosh \chi,
\end{gathered}
$$

where the velocities $v_{1}=p_{1} / m$ and $v_{2}=p_{2} / m$ determine the scattering plane. In this system the instanton position is given by $z_{0}=\left(z_{4}, z_{3}, z_{\perp}\right)$.

However, due to exponentially large oscillations at large $\chi$ that occur during integration over instanton position, it is not easy to use the closed form for the phases (6.5) in general case and for the Gaussian profile (9.1), in particular. We have to note that these complications do not arise in the case of high-energy elastic quark-quark scattering considered in [53]. In the latter case, the angle dependence simply factorizes from the integrand in the asymptotic regime (see the next Section).

In order to cancel exponentially large oscillations, we need to integrate first over the instanton position $z_{0}$. For this purpose, by using the explicit form (9.1) we expand the expressions (6.10) in powers of the phases (6.5) and change the order of integrations:

$$
w_{c}^{I}(\chi)=\sum_{n=1}^{\infty} \sum_{m=1}^{\infty}(-1)^{n+m} \frac{\left\langle\alpha_{1}^{2 n} \alpha_{2}^{2 m}\right\rangle(\chi)}{(2 n) !(2 m) !}, \quad w_{s}^{I}(\chi)=\sum_{n=0}^{\infty} \sum_{m=0}^{\infty}(-1)^{n+m} \frac{\left\langle\alpha_{1}^{2 n+1} \alpha_{2}^{2 m+1}\right\rangle(\chi)}{(2 n+1) !(2 m+1) !},
$$

where

$$
\left\langle\alpha_{1}^{2 n} \alpha_{2}^{2 m}\right\rangle(\chi)=\int_{0}^{\infty} \prod_{i=1}^{2 n} d \lambda_{i} \int_{0}^{\infty} \prod_{j=1}^{2 m} d \lambda_{j}^{\prime} \int d^{4} z_{0} s^{2 n}\left(v_{1}, z_{0}\right) s^{2 m}\left(v_{2}, z_{0}\right) e^{-\left[\left(v_{1} \lambda_{i}-z_{0}\right)^{2}+\left(v_{2} \lambda_{j}^{\prime}+z_{0}\right)^{2}\right]},
$$

\footnotetext{
${ }^{5}$ This average is infinite in the regular gauges. Thus, the singular gauge realizes the minimal Landau gauge for the instantons.

${ }^{6}$ In these expressions and below we make formally analytical continuation to the Minkowski space that is inverse to the transformation (6.1).
} 
and

$$
\begin{aligned}
\left\langle\alpha_{1}^{2 n+1} \alpha_{2}^{2 m+1}\right\rangle(\chi)= & \int_{0}^{\infty} \prod_{i=0}^{2 n+1} d \lambda_{i} \int_{0}^{\infty} \prod_{j=0}^{2 m+1} d \lambda_{j}^{\prime} \int d^{4} z_{0} s^{2 n}\left(v_{1}, z_{0}\right) s^{2 m}\left(v_{2}, z_{0}\right) s_{12}^{2}\left(z_{0}\right) \\
& \cdot e^{-\left[\left(v_{1} \lambda_{i}-z_{0}\right)^{2}+\left(v_{2} \lambda_{j}^{\prime}+z_{0}\right)^{2}\right]}
\end{aligned}
$$

Note, that the integrands possess the symmetry with respect to the change of variables

$$
z_{3} \rightarrow z_{3} \cosh \chi+i z_{4} \sinh \chi, \quad z_{4} \rightarrow z_{4} \cosh \chi-i z_{3} \sinh \chi
$$

Above we introduced the notation for the color spin correlation factor (its normalized definition given above in (6.11))

$$
s_{12}^{2}\left(z_{0}\right)=z_{3}\left(z_{3} \cosh \chi-i z_{4} \sinh \chi\right)+z_{\perp}^{2} \cosh \chi .
$$

Let us further make the change of variables $\lambda$ by introducing the total and partial lengths

$$
\left\{\lambda_{i}\right\}_{N} \rightarrow\left\{L=\sum_{i=1}^{N} \lambda_{i}, x_{i}=\frac{\lambda_{i}}{L}\right\}, \quad\left\{\lambda_{i}^{\prime}\right\}_{M} \rightarrow\left\{L^{\prime}=\sum_{j=1}^{M} \lambda_{j}^{\prime}, y_{j}=\frac{\lambda_{i}^{\prime}}{L^{\prime}}\right\}
$$

with new measures given by

$$
\int_{0}^{\infty} d L L^{N-1} \int d\{x\}_{N}, \quad \int_{0}^{\infty} d L^{\prime} L^{\prime M-1} \int d\{y\}_{M},
$$

where $\int d\{x\}_{N}=\int_{0}^{1} d x_{1} \ldots \int_{0}^{1-x_{1}-\ldots-x_{N-2}} d x_{N-1}$.

After these transformations we come to the general expression for the phase averages (9.8) and (9.9) with arbitrary $M$ and $N$

$$
\left\langle\alpha_{1}^{N} \alpha_{2}^{M}\right\rangle(\chi)=\int_{0}^{\infty} d L \int_{0}^{\infty} d L^{\prime} e^{-\frac{2}{N+M} L L^{\prime} \cosh \chi} G_{N, M}\left(L, L^{\prime}, \chi\right) F_{N, M}(L) F_{M, N}\left(L^{\prime}\right),
$$

with definitions

$$
\begin{gathered}
F_{N, M}(L)= \\
=L^{N-1} \int d\{x\}_{N} \exp \left\{-\frac{1}{N+M}\left[L^{2}+(N+M-2) \sum_{i}^{N} \lambda_{i}^{2}-4 \sum_{i>j}^{N} \lambda_{i} \lambda_{j}\right]\right\}, \\
G_{N, M}\left(L, L^{\prime}, \chi\right)=\int d^{4} z_{0} s^{2 n}\left(v_{1}, z_{0}\right) s^{2 m}\left(v_{2}, z_{0}\right) s_{12}^{2 \eta}\left(z_{0}\right) e^{-(N+M)\left(z_{\perp}^{2}+z_{4}^{\prime 2}+z_{3}^{\prime 2}\right)}
\end{gathered}
$$

where $z_{4}^{\prime}=z_{4}-\frac{L-L^{\prime} \cosh \chi}{N+M}$ and $z_{3}^{\prime}=z_{3}+\frac{i L^{\prime} \sinh \chi}{N+M}$. The definition for $F_{M, N}\left(L^{\prime}\right)$ is similar to one of $F_{N, M}(L)$, the power $\eta$ in $G_{N, M}\left(L, L^{\prime}, \chi\right)$ is equal to one for $w_{s}^{I}(\chi)$ and to zero for $w_{c}^{I}(\chi)$.

In principle, the integral in $G_{N, M}\left(L, L^{\prime}, \chi\right)$ may be done analytically for the Gaussian profile in arbitrary order of expansion for any finite values of $\chi$. In this case, after the 
$d^{4} z_{0}$ integration there remain no complex valued variables anymore and the Euclidean (transverse) and Minkowski (longitudinal in $\chi$ ) dependencies get factorized. But in practice only a few first terms can be analyzed. Nevertheless, as we will see below, in the large $\chi$ limit it is possible to make the partial summation of the double sums in the expressions (9.7). Let us demonstrate these statements in more detail.

Consider the lowest order one-loop and two-loop contributions given by (Fig. đ)

$$
w^{I}(\chi)=\frac{2 n_{c}}{3}\left(-\left.\left\langle\alpha_{1} \alpha_{2}\right\rangle(\chi)\right|_{S}+\left.\frac{1}{6}\left\langle\alpha_{1}^{3} \alpha_{2}\right\rangle(\chi)\right|_{S}+\left.\frac{1}{6}\left\langle\alpha_{1} \alpha_{2}^{3}\right\rangle(\chi)\right|_{S}+\left.\frac{1}{4}\left\langle\alpha_{1}^{2} \alpha_{2}^{2}\right\rangle(\chi)\right|_{S}+\ldots\right),
$$

where $\left.\left\langle\alpha_{1}^{M} \alpha_{2}^{N}\right\rangle(\chi)\right|_{S}=\left\langle\alpha_{1}^{M} \alpha_{2}^{N}\right\rangle(\chi)-\left\langle\alpha_{1}^{M} \alpha_{2}^{N}\right\rangle(0)$. The simplest one-loop diagram (Fig. 4a) corresponds to the first term in (9.17) $(N=M=1$ term in Eq. (9.14)). In that case, the integrals may be done analytically (by using the Eqs. (13.3) and (13.4) ) and the final result reduces to the weak field expression (8.5) with the function $\Delta_{1}$ given by

$$
\Delta_{1}^{G}\left(x^{2}\right)=-\frac{\pi^{2} \rho_{c}^{4}}{4} e^{-x^{2} / 2 \rho_{c}^{2}}
$$

Thus, in the lowest order we have the result $\left(f=\pi^{2} n_{c} \rho_{c}^{4}\right)$

$$
w_{G}^{(1,1)}(\chi)=-\frac{f}{12} h(\chi)
$$

and its small and large $\chi$ behavior

$$
w_{G}^{(1,1)}(\chi \rightarrow 0)=-\frac{f}{36} \chi^{2}, \quad w_{G}^{(1,1)}(\chi \rightarrow \infty)=-\frac{f}{12} \chi .
$$

It is important to note, that due to screening of instantons at large distances the result (9.18) is IR-finite contrary to the case of the pure instanton solution in (8.7).

If one neglected the color spin factors in (9.16), one would get another dependence on the scattering angle

$$
\begin{aligned}
w_{G s}^{(1,1)}(\chi) & =-\frac{f}{6}\left(1-\frac{\chi}{\sinh \chi}\right), \\
w_{G s}^{(1,1)}(\chi \rightarrow 0) & =-\frac{f}{36} \chi^{2}, \quad w_{G s}^{(1,1)}(\chi \rightarrow \infty)=-\frac{f}{6},
\end{aligned}
$$

thus getting the asymptotic which is not enhanced by the large factor $\chi$.

The rest of Eq. (9.17) corresponds to the two-loop diagrams shown in Fig. (四 $\mathrm{b}$ ) $(N=1, M=3$ in (9.14) $)$ and in Fig. 4tc $(N=M=2$ in (9.14) $)$. Then, the functions $F_{N, M}(L)$ and $G_{N, M}\left(L, L^{\prime}, \chi\right)$ becomes

$$
\begin{aligned}
& F_{1,3}(L)=\exp \left(-\frac{3}{4} L^{2}\right), \quad F_{2,2}(L)=\sqrt{\frac{\pi}{2}} \exp \left(-\frac{1}{4} L^{2}\right) \operatorname{erf}\left(\frac{L}{\sqrt{2}}\right), \\
& F_{3,1}(L)=L^{2} \int_{0}^{1} d x \int_{0}^{1-x} d x^{\prime} e^{-\frac{1}{4} L^{2}\left[3+8\left(x^{2}+x^{\prime 2}+x x^{\prime}-x-x^{\prime}\right)\right]},
\end{aligned}
$$




$$
\begin{aligned}
& G_{2,2}\left(L, L^{\prime}, \chi\right)=\int d^{4} z_{0} s^{2}\left(v_{1}, z_{0}\right) s^{2}\left(v_{2}, z_{0}\right) e^{-4\left(z_{\perp}^{2}+t^{\prime 2}+z_{3}^{\prime 2}\right)}= \\
& =\frac{\pi^{2}}{2^{12}}\left[L^{\prime 2} \sinh ^{2} \chi\left(L^{2} \sinh ^{2} \chi-6\right)-8 L L^{\prime} \sinh ^{2} \chi \cosh \chi-6 L^{2} \sinh ^{2} \chi+52+8 \cosh ^{2} \chi\right] \\
& G_{1,3}\left(L, L^{\prime}, \chi\right)=\int d^{4} z_{0} s^{2}\left(v_{1}, z_{0}\right) s_{12}^{2}\left(z_{0}\right) e^{-4\left(z_{\perp}^{2}+t^{\prime 2}+z_{3}^{\prime 2}\right)}= \\
& =\frac{\pi^{2}}{2^{12}}\left[-L L^{\prime 3} \sinh ^{4} \chi+10 L^{\prime} \sinh ^{2} \chi\left(L^{\prime} \cosh \chi+L\right)-60 \cosh \chi\right] .
\end{aligned}
$$

We compare the full result and the one-loop and two-loop approximations to it at small $\chi$ in Fig. 5 .

In Fig. 6 we also present the results corresponding to the calculations without color spin factors, $s\left(v_{i}, z_{0}\right)$. In the latter case, the coordinate integral $\int d^{4} z_{0}$ in (9.16) may be performed easily with the result

$$
G_{N, M}^{s}\left(L, L^{\prime}, \chi\right)=\frac{\pi^{2}}{(M+N)^{2}} .
$$

The next order calculations may be done in a similar way. Finally, it may be shown that in the limit of large scattering angle $\chi$ the asymptotics reads $w_{G s}^{I}(\chi \rightarrow \infty) \sim$ const. Thus, in this case one has weaker asymptotics than the asymptotics with color spin factor included.

In the following, we are interested in the limit $\chi \rightarrow \infty$, where the coefficient of $\chi$ is free of the light-cone singularities and therefore it has a well defined limit for the onshell quark momenta, $p_{1}^{2}=p_{2}^{2}=0$. To find this asymptotics, we take into account that $F_{N, M}(L \rightarrow 0) \neq 0$ and $G_{N, M}\left(L, L^{\prime}\right)$ are polynomials in $L$ and $L^{\prime}$. With these properties and in the large $\chi$ limit, the $L$ and $L^{\prime}$ integrations may be performed analytically

$$
\begin{aligned}
& \lim _{\chi \rightarrow \infty} \int_{0}^{\infty} d L \int_{0}^{\infty} d L^{\prime}\left(L L^{\prime}\right)^{n} e^{-\alpha L L^{\prime} \cosh \chi} F_{N, M}(L) F_{M, N}\left(L^{\prime}\right)= \\
& =\frac{n ! \chi}{(\alpha \cosh \chi)^{n+1}} F_{N, M}(0) F_{M, N}(0), \\
& \lim _{\chi \rightarrow \infty} \int_{0}^{\infty} d L \int_{0}^{\infty} d L^{\prime}\left(L L^{\prime}\right)^{n} L^{m} e^{-\alpha L L^{\prime} \cosh \chi} F_{N, M}(L) F_{M, N}\left(L^{\prime}\right)= \\
& =\frac{n !}{(\alpha \cosh \chi)^{n+1}} F_{M, N}(0) \int d L L^{m-1} F_{N, M}(L) .
\end{aligned}
$$

The important result is that the only diagonal terms with equal powers of $L$ and $L^{\prime}$ provide the leading in $\chi$ asymptotics.

Let us consider the contribution to the asymptotics from the terms with $m=0$ and arbitrary $n$ of $w_{s}^{I}(\chi)$ (Fig. 7a). This contribution is reduced to the element

$$
\begin{aligned}
& \left\langle\alpha_{1}^{2 n+1} \alpha_{2}^{1}\right\rangle=-\cosh \chi \int_{0}^{\infty} d L L^{2 n} \int_{0}^{\infty} d L^{\prime} \int d\{x\}_{2 n+1} e^{-L^{2} \sum_{i}^{2 n} x_{i}^{2}-L^{\prime 2}} . \\
& \cdot \int d^{4} z_{0}\left(z_{3}^{2}-i z_{3} z_{4} \tanh \chi+z_{\perp}^{2}\right)\left(z_{3}^{2}+z_{\perp}^{2}\right)^{n} e^{-\left[2(n+1) z_{0}^{2}-2 z_{4}\left(L-L^{\prime} \cosh \chi\right)+2 i z_{3} L^{\prime} \sinh \chi\right]} .
\end{aligned}
$$

The $z_{0}$ integration gives (see Eqs. (13.7) and (13.8)

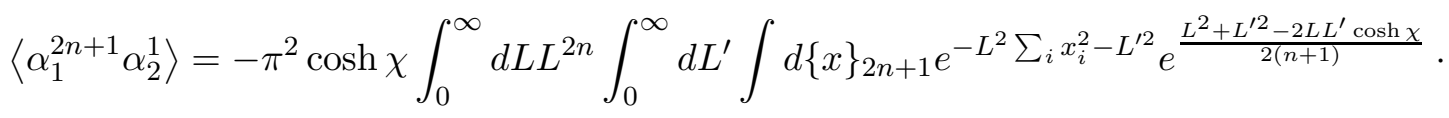




$$
\begin{aligned}
& \cdot \sum_{k=0}^{n} C_{k}^{n} \frac{k !}{[2(n+1)]^{k+2}}\left[\frac{k+1}{2(n+1)}\left(\frac{i L^{\prime} \sinh \chi}{2(n+1)}\right)^{2(n-k)} \sum_{s=0}^{n-k} \bar{C}_{s}^{2(n-k)} y^{s}+\right. \\
& +\left(\frac{i L^{\prime} \sinh \chi}{2(n+1)}\right)^{2(n-k+1)} \sum_{s=0}^{n-k+1} \bar{C}_{s}^{2(n-k+1)} y^{s}-\left(\frac{i L^{\prime} \sinh \chi}{2(n+1)}\right)^{2(n-k+1)} \sum_{s=0}^{n-k} \bar{C}_{s}^{2(n-k)+1} y^{s}+ \\
& \left.+\frac{i L \tanh \chi}{2(n+1)}\left(\frac{i L^{\prime} \sinh \chi}{2(n+1)}\right)^{2(n-k)+1} \sum_{s=0}^{n-k} \bar{C}_{s}^{2(n-k)+1} y^{s}\right]
\end{aligned}
$$

where we introduce notation

$$
y=\frac{2(n+1)}{\left(i L^{\prime} \sinh \chi\right)^{2}}
$$

The Eq. (9.28) is still a general expression. To find its large- $\chi$ limit, we need to analyze, according to 9.25$)$, the coefficients of the maximal powers of the diagonal terms $\left(L L^{\prime}\right)^{2 n}$. In the first and fourth terms in the brackets of (9.28), the only terms with $k=s=0$ provide the leading $\chi$ asymptotics and the other one gives the subleading contribution. The second and third terms in the brackets give dominant asymptotics if $k+s \leq 1$. Their sum provides the leading $\chi$-asymptotics, while the terms of higher powers in $\chi \cosh \chi$ are canceled. Keeping only leading terms in the Eq. (9.28), one gets

$$
\begin{aligned}
& \left.\left\langle\alpha_{1}^{2 n+1} \alpha_{2}^{1}\right\rangle\right|_{\chi \rightarrow \infty}=-\frac{\pi^{2} \cosh \chi}{16(n+1)^{3}} \int_{0}^{\infty} d L L^{2 n} \int_{0}^{\infty} d L^{\prime} \int d\{x\}_{2 n+1} e^{-L^{2} \sum_{i} x_{i}^{2}-L^{\prime 2}} . \\
& \cdot e^{\frac{\left[L^{2}+L^{\prime 2}-2 L L^{\prime} \cosh \chi\right.}{2(n+1)}}\left(\frac{i L^{\prime} \sinh \chi}{2(n+1)}\right)^{2 n}\left(2 n+3-\frac{L L^{\prime} \sinh ^{2}(\chi)}{(n+1) \cosh (\chi)}\right) .
\end{aligned}
$$

As $\chi \rightarrow \infty$, all integrals can be done analytically by using Eq. (9.25):

$$
\left.\left\langle\alpha_{1}^{2 n+1} \alpha_{2}^{1}\right\rangle\right|_{\chi \rightarrow \infty}=\frac{(-1)^{n+1} \pi^{2}(2 n) !}{2^{4 n+3}(n+1)^{2}} \chi .
$$

Now, let us consider the contribution to the asymptotics of $w_{c}^{I}(\chi)$ from the terms with $m=1$ and arbitrary $n$ (Fig. 7 $\mathrm{b}$ ):

$$
\begin{aligned}
& \left\langle\alpha_{1}^{2 n} \alpha_{2}^{2}\right\rangle=\cosh ^{2} \chi \int_{0}^{\infty} d L L^{2 n-1} \int_{0}^{\infty} d L^{\prime} L^{\prime} \int d\{x\}_{2 n} \int_{0}^{1} d y e^{-L^{2} \sum_{i} x_{i}^{2}-L^{\prime 2}\left(y^{2}+(1-y)^{2}\right)} . \\
& \cdot \int d^{4} z_{0}\left(\frac{z_{\perp}^{2}}{\cosh ^{2} \chi}+z_{3}^{2}+2 i z_{3} z_{4} \tanh \chi-z_{4}^{2} \tanh ^{2} \chi\right) . \\
& \cdot \sum_{k=0}^{n} C_{k}^{n} z_{\perp}^{2 k} z_{3}^{2(n-k)} e^{-\left[2(n+1) z_{0}^{2}+2 z_{4}\left(L-L^{\prime} \cosh \chi\right)+2 i z_{3} \sinh \chi\right]} .
\end{aligned}
$$

By using the table integrals Eq.(13.7) and (13.8), the Gaussian integration over $z_{0}$ yields

$$
\begin{aligned}
& \left\langle\alpha_{1}^{2 n} \alpha_{2}^{2}\right\rangle=\pi^{2} \int_{0}^{\infty} d L \int_{0}^{\infty} d L^{\prime}\left(L L^{\prime}\right) L^{2(n-1)} \int d\{x\}_{2 n} \int_{0}^{1} d y e^{-L^{2} \sum_{i} x_{i}^{2}-L^{\prime 2}\left(y^{2}+(1-y)^{2}\right)} . \\
& \cdot e^{\frac{L^{2}+L^{\prime 2}-2 L L^{\prime} \cosh \chi}{2(n+1)}} \sum_{k=0}^{n} C_{k}^{n} \frac{k !}{[2(n+1)]^{n+3}}\left(\frac{L^{\prime 2} \sinh ^{2} \chi}{2(n+1)}\right)^{(n-k)} .
\end{aligned}
$$




$$
\begin{aligned}
& {\left[\sum_{s=0}^{n-k} \bar{C}_{s}^{2(n-k)} y^{s}\left(-\frac{L^{2} \sinh ^{2} \chi}{2(n+1)}+\frac{3}{2}+k+s \cosh ^{2} \chi\right)-\right.} \\
& \left.-\sum_{s=0}^{n-k-1} \bar{C}_{s}^{2(n-k)}(n-k-s) y^{s+1} L L^{\prime} \sinh ^{2} \chi \cosh \chi\right]
\end{aligned}
$$

with $y$ given by Eq.(9.29). Again, the large $\chi$ limit of this expression stems from the diagonal terms $\left(L L^{\prime}\right)^{2 n}$

$$
\begin{aligned}
& \left.\left\langle\alpha_{1}^{2 n} \alpha_{2}^{2}\right\rangle\right|_{\chi \rightarrow \infty}= \\
& \frac{(-1)^{n-1} \pi^{2} \cosh \chi}{[2(n+1)]^{2(n+2)}} \int_{0}^{\infty} d L \int_{0}^{\infty} d L^{\prime}\left(L L^{\prime} \sinh \chi\right)^{2 n-1} \int d\{x\}_{2 n} \int_{0}^{1} d y e^{-L^{2} \sum_{i} x_{i}^{2}-L^{\prime 2}\left(y^{2}+(1-y)^{2}\right) .} \\
& \cdot e^{\frac{L^{2}+L^{\prime 2}-2 L L^{\prime} \cosh \chi}{2(n+1)}}\left[n(n+1)(2 n-1)-4 n(n+1) L L^{\prime} \sinh \chi+\left(L L^{\prime} \sinh \chi\right)^{2}\right] .
\end{aligned}
$$

After integration one finds that the coefficient of the leading asymptotics is equal to zero, and therefore

$$
\left.\left\langle\alpha_{1}^{2 n} \alpha_{2}^{2}\right\rangle\right|_{\chi \rightarrow \infty}=\text { const } .
$$

Moreover, it is possible to show that further leading asymptotic terms appear only if $n \geq 3$ and $m \geq 3$, but they are highly suppressed numerically.

Thus from (6.9), (9.7) and (9.31), we find that the leading correction to the quark form factor is given by

$$
w_{G}\left(q^{2}\right)=-\sum_{n=0}^{\infty} \frac{1}{16^{n}(n+1)^{2}(2 n+1)} \cdot \frac{f}{12} \ln q^{2}=-1.0053 \frac{f}{12} \ln q^{2},
$$

that means that the weak field limit $(9.20)$ is a good approximation for the Gaussian profile function (9.1). This estimate of the logarithmic coefficient- $B_{G} \approx 0.01$, is compatible with the estimate 8.22 ) obtained in the single instanton approximation.

We have to comment that in the general (non-Gaussian) case the weak-field limit may deviate from the exact result. Nevertheless, it is reasonable to expect that by taking the instanton solution in the singular gauge which concentrate the field at small distances and allows us to prove the exponentiation theorem for the Wilson loop in the instanton field [46], one gets a good numerical estimate of the full effect. Thus, the resulting diminishing of the instanton contributions with respect to the perturbative result appears to be reasonable output. The analysis of all-order instanton contribution performed in the last part of this Section for a Gaussian profile function shows that the weak field approximation can be justified, but an additional investigation of this problem is required.

\section{Instanton model of Pomeron}

Soft hadronic collisions are described successfully within the Regge phenomenology, with the Pomeron exchange being dominating at high energy. The Pomeron is considered as an effective exchange in the $t$ channel by the object with vacuum quantum numbers and with positive charge parity $C=+1$. That is why the idea that the nontrivial structure 
of the QCD vacuum is relevant in describing its mechanism. To illustrate this idea, let us consider the high energy diffractive quark-quark scattering, where there is a hope that for small momentum transfer the nonperturbative effects give dominant contribution. One of the simplest models of the Pomeron is based on the use of exchange by two nonperturbative gluons. The nonperturbative part of the gluon propagator is given by (in the Landau gauge)

$$
\left\langle 0\left|: A_{\mu}^{a}(x) A_{\nu}^{b}(0):\right| 0\right\rangle=\frac{1}{g^{2}} \frac{\delta^{a b}}{N_{c}^{2}-1} \int \frac{d^{4} k}{(2 \pi)^{4}} \mathrm{e}^{-i k x}\left(g^{\mu \nu}-\frac{k^{\mu} k^{\nu}}{k^{2}}\right) G_{n p}\left(k^{2}\right) .
$$

In the Abelian gauge model considered originally by Landshoff and Nachtmann [16], the nonperturbative gluon propagator (without a color factor) $G_{n p}\left(k^{2}\right)$ is related to the correlation function describing the gauge invariant gluon field strength correlator (nonlocal gluon condensate). In general non-Abelian case this correlator has the form

$$
\begin{gathered}
\left\langle 0\left|: G_{\mu \nu}(x) \mathcal{P} \exp \left[i g \int_{0}^{x} d z^{\alpha} A_{\alpha}(z)\right] G_{\rho \sigma}(0):\right| 0\right\rangle= \\
=\int \frac{d^{4} k}{(2 \pi)^{4}} \mathrm{e}^{-i k x}\left[\left(D_{0}\left(k^{2}\right)+D_{1}\left(k^{2}\right)\right) k^{2}\left(g_{\mu \rho} g_{\nu \sigma}-g_{\mu \sigma} g_{\nu \rho}\right)+\right. \\
\left.+D_{1}\left(k^{2}\right)\left(k_{\mu} k_{\rho} g_{\nu \sigma}-k_{\mu} k_{\sigma} g_{\nu \rho}+k_{\nu} k_{\sigma} g_{\mu \rho}-k_{\nu} k_{\rho} g_{\mu \sigma}\right)\right],
\end{gathered}
$$

where the first tensor structure is called non-Abelian part and the second one is Abelian part. Indeed, in the Abelian gauge model without monopoles $D_{0}\left(k^{2}\right) \equiv 0$, and $D_{1}\left(k^{2}\right)=$ $G_{n p}\left(k^{2}\right)$. It is this property that has been used in [16] to relate the Pomeron properties to the value of the gluon condensate.

However, in the non-Abelian model one has the opposite situation. Really, for the QCD instantons we find [66, 67] $D_{1}\left(k^{2}\right) \equiv 0$ and $D_{0}\left(k^{2}\right)$ is nonzero. In the realistic model of the QCD vacuum, where the interaction with vacuum fields of large scale, $R$, is important, the instanton ceases to be exact solution of the equations of motion, but the so-called constrained instanton approximate solution (CI) can be constructed [67]. It has been shown that the constrained instanton has exponentially decreasing asymptotics at large distances $(\sim R)$. The constrained instanton has topological number \pm 1 like an instanton; however, it is not self-dual field. Thus, in the realistic QCD, the small non-zero part of $D_{1}\left(k^{2}\right)$ appears. Very similar results have been found in the lattice simulations of the gluon field strength correlator [72].

Thus, within the non-Abelian models there is no direct connection of the gluon propagator to the gluon field strength correlator. So, let us explicitly consider the instanton part of the gluon propagator. The Fourier transform of the instanton field is defined as

$$
\widetilde{A}_{\mu}^{a}(k)=\frac{1}{g} \eta_{\mu \nu}^{a} k_{\nu} \widetilde{\phi}\left(k^{2}\right)
$$

where

$$
\widetilde{\phi}\left(k^{2}\right)=\frac{4 \pi^{2} i}{k^{2}} \int_{0}^{\infty} d x x^{3} J_{2}(|k| x) \varphi\left(x^{2}\right),
$$


and $J_{2}(z)$ is the Bessel function. The function $\widetilde{\phi}\left(k^{2}\right)$ is related to the function $\widetilde{\varphi}\left(k^{2}\right)$ defined in (8.2) by

$$
\widetilde{\phi}\left(k^{2}\right)=-2 i \frac{\partial \widetilde{\varphi}\left(k^{2}\right)}{\partial k^{2}} .
$$

The explicit form of the Fourier transform of the pure instanton solution is well known (in the singular gauge)

$$
\widetilde{\phi}^{I}\left(k^{2}\right)=i \frac{(4 \pi)^{2}}{k^{4}}\left[1-\frac{\left(\rho_{c} k\right)^{2}}{2} K_{2}\left(\rho_{c} k\right)\right], \quad \widetilde{\phi}^{I}\left(k^{2}\right)= \begin{cases}\frac{i(2 \pi)^{2} \rho_{c}^{2}}{k^{2}}, & k^{2} \rightarrow 0, \\ \frac{i(4 \pi)^{2}}{k^{4}}, & k^{2} \rightarrow \infty .\end{cases}
$$

The (constrained) instanton profile may be chosen in the form (c.f. Eq. (3.6))

$$
\varphi_{C I}(x)=\frac{\rho_{C I}^{2}\left(x^{2}\right)}{x^{2}\left(x^{2}+\rho_{C I}^{2}\left(x^{2}\right)\right)}, \quad \rho_{C I}^{2}\left(x^{2}\right)=2\left(\frac{\rho_{c}}{R}\right)^{2} x^{2} K_{2}\left(\frac{|x|}{R}\right),
$$

where $K_{2}(z)$ is the modified Bessel function. The constrained solution saves its form at short distances, but changes it at large ones:

$$
\widetilde{\phi}_{C I}\left(k^{2}\right)=\left\{\begin{array}{cc}
\frac{i \pi^{2}}{4} R^{4} I_{C I}, & k^{2} \rightarrow 0, \\
\frac{i(4 \pi)^{2}}{k^{4}}, & k^{2} \rightarrow \infty
\end{array}\right.
$$

where the constant $I_{C I}$ is given by

$$
I_{C I}=\int_{0}^{\infty} d u u^{2} \varphi_{C I}\left(u R^{2}\right) .
$$

Now the Fourier transform of the single instanton contribution to the gluon propagator (10.1) becomes

$$
G_{n p}\left(k^{2}\right)=-4 n_{c} k^{2} \widetilde{\phi}^{2}\left(k^{2}\right) .
$$

Thus, we see that the nonperturbative gluon propagator (10.1), (10.10) and gluon field strength correlator (10.2) are quite different functions, and the relation between them that is valid in the Abelian gauge model is destroyed in the non-Abelian case.

From (10.6) and (10.8) one gets the asymptotics of the instanton part of the gluon propagator

$$
G^{I}\left(k^{2}\right)=\left\{\begin{array}{c}
(2 \pi)^{4} n_{c} \rho_{c}^{4} k^{-2}, \\
(4 \pi)^{4} n_{c} k^{-6}, \\
k^{2} \rightarrow 0
\end{array} \quad, \quad k^{C I}\left(k^{2}\right)=\left\{\begin{array}{cc}
\frac{\pi^{4} n_{c} R^{8}}{16} I_{C I}^{2} k^{2}, & k^{2} \rightarrow 0 \\
(4 \pi)^{4} n_{c} k^{-6}, & k^{2} \rightarrow \infty
\end{array}\right.\right.
$$

Calculating (in a very similar way as in the Landshoff-Nachtmann model) the invariant $\mathcal{T}$-matrix element of the quark-quark scattering at large energy, $s$, and small transferred momentum, $t$, we get

$$
\left.\left\langle q_{k}\left(p_{3}\right) q_{l}\left(p_{4}\right)|\mathcal{T}| q_{m}\left(p_{1}\right) q_{n}\left(p_{2}\right)\right\rangle\right|_{s \rightarrow \infty} \rightarrow i I(t) \quad \bar{u}\left(p_{3}\right) \gamma^{\mu} u\left(p_{1}\right) \bar{u}\left(p_{4}\right) \gamma^{\mu} u\left(p_{2}\right) \delta_{k m} \delta_{l n}
$$

with

$$
I(t)=\frac{1}{72} \int \frac{d \boldsymbol{k}_{\perp}}{(2 \pi)^{2}} G\left[\left(\boldsymbol{k}_{\perp}+\frac{1}{2} \boldsymbol{q}_{\perp}\right)^{2}\right] G\left[\left(\boldsymbol{k}_{\perp}-\frac{1}{2} \boldsymbol{q}_{\perp}\right)^{2}\right]
$$


where

$$
s=\left(p_{1}+p_{2}\right)^{2}=2 m^{2}(1+\cosh \chi), \quad t=-\left(p_{3}-p_{1}\right)^{2}=-q_{\perp}^{2},
$$

$G\left(k^{2}\right)$ is defined in (10.10) with $k^{2} \rightarrow \boldsymbol{k}_{\perp}^{2}$. Except of the numerical coefficient, this expression is in agreement with the Landshoff-Nachtmann formula. This agreement is due to the specific features of the instanton induced interaction. From the infrared behavior of the instanton induced propagator (10.11), it is clear that $I(0)$ is infinite for the pure instanton solution (10.6), but it is finite for the constrained instanton solution (10.7). This fact, also noted recently in [68], was one of the arguments to construct the constrained instanton solution. The form of $I(t)$ is presented in Fig. 9 for the constrained instanton configuration.

It is possible to show that the result $(10.12)$ is the weak field limit of the more general expression. Indeed, the quark-quark scattering amplitude may be expressed in terms of the vacuum average of the gauge invariant path ordered Wilson integral [17, 18]

$$
T_{m n}^{k l}(s, t)=-2 i s \int d^{2} \boldsymbol{b}_{\perp} \mathrm{e}^{i b_{\perp} q} W_{m n}^{k l}\left(\gamma, \boldsymbol{b}_{\perp}^{2}\right),
$$

where the Wilson line function $W_{m n}^{k l}$ is given by

$$
W_{m n}^{k l}\left(\gamma, b_{\perp}^{2}\right)=\left\langle 0\left|\mathcal{P} \exp \left\{i g \int_{C_{q q}} d x_{\mu} \hat{A}_{\mu}(x)\right\}\right| 0\right\rangle_{m n}^{k l} .
$$

In Eq. (10.15), the corresponding integration path goes along the closed contour $C_{q q}$ : two infinite lines separated by the transverse distance $\boldsymbol{b}_{\perp}$ and having relative scattering angle $\chi$. We parameterize the integration path $C_{\chi}=\left\{z_{\mu}(\lambda) ; \lambda=[-\infty, \infty]\right\}$ as follows

$$
z_{\mu}(\lambda)=\left\{\begin{array}{cc}
v_{1} \lambda, & -\infty<\lambda<\infty, \\
v_{2} \lambda+b_{\perp}, & -\infty<\lambda<\infty,
\end{array}\right.
$$

with $\left(v_{1} v_{2}\right)=\cosh \chi$ and $\boldsymbol{b}_{\perp}$ being the impact parameter.

By making the steps similar to the previous Section, one arrives to the expression for the Wilson line function (c.f. Eq. (6.10) ) (see Fig. 8)

$$
\begin{aligned}
& W\left(\gamma, b_{\perp}^{2}\right)=n_{c}\left\{\frac{4}{9} w_{c}\left(\gamma, b_{\perp}^{2}\right)(\mathbb{I} \times \mathbb{I})+\frac{1}{8}\left[\frac{1}{3} w_{c}\left(\gamma, b_{\perp}^{2}\right)+w_{s}\left(\gamma, b_{\perp}^{2}\right)\right]\left(\lambda^{A} \times \lambda^{A}\right)\right\} \\
& w_{c}\left(\gamma, b_{\perp}^{2}\right)=\int d^{4} z_{0}\left[\cos \alpha\left(v_{1}, z_{0}\right)-1\right]\left[\cos \alpha\left(v_{2}, z_{0}\right)-1\right], \\
& w_{s}\left(\gamma, b_{\perp}^{2}\right)=-\int d^{4} z_{0}\left(\hat{n}_{1}^{a} \hat{n}_{2}^{a}\right) \sin \alpha\left(v_{1}, z_{0}\right) \sin \alpha\left(v_{2}, z_{0}\right),
\end{aligned}
$$

where the color correlation factor is

$$
\hat{n}_{1}^{a} \hat{n}_{2}^{a}=\frac{\left(v_{1} v_{2}\right)\left(z_{0}, z_{0}-b_{\perp}\right)-\left(v_{1} z_{0}\right)\left(v_{2} z_{0}\right)}{s\left(v_{1}, z_{0}\right) s\left(v_{2}, z_{0}\right)} .
$$

The phases are defined as

$$
\begin{aligned}
& \alpha\left(v_{1}, z_{0}\right)=s\left(v_{1}, z_{0}\right) \int_{-\infty}^{\infty} d \lambda \varphi\left[\left(z_{0}+v_{1} \lambda\right)^{2} ; \rho\right] \\
& \alpha\left(v_{2}, z_{0}\right)=s\left(v_{2}, z_{0}\right) \int_{-\infty}^{\infty} d \lambda \varphi\left[\left(z_{0}-v_{2} \lambda-b_{\perp}\right)^{2} ; \rho\right] .
\end{aligned}
$$


with

$$
s^{2}\left(v_{1}, z_{0}\right)=z_{0}^{2}-\left(v_{1} z_{0}\right) ; \quad s^{2}\left(v_{2}, z_{0}\right)=\left(z_{0}-b_{\perp}\right)^{2}-\left(v_{2} z_{0}\right) .
$$

By means of the change of variables $\left(z_{4} \cos \gamma-z_{3} \sin \gamma\right) \rightarrow z_{4}$, the energy dependence is trivially factorized

$$
w_{c}\left(\gamma, b_{\perp}^{2}\right) \rightarrow \frac{1}{\sin \gamma} w_{c}\left(\gamma=\pi / 2, b_{\perp}^{2}\right), \quad w_{s}\left(\gamma, b_{\perp}^{2}\right) \rightarrow \cot \gamma w_{s}\left(\gamma=\pi / 2, b_{\perp}^{2}\right) .
$$

At $\gamma=\pi / 2$ the above definitions are reduced to

$$
\begin{aligned}
& s^{2}\left(v_{1}, z_{0}\right) \rightarrow s_{1}^{2}=z_{3}^{2}+z_{\perp}^{2}, \quad s^{2}\left(v_{2}, z_{0}\right) \rightarrow s_{2}^{2}=z_{4}^{2}+(z-b)_{\perp}^{2}, \\
& \hat{n}_{1}^{a} \hat{n}_{2}^{a}(\gamma=\pi / 2)=\frac{z_{\perp}^{2}-\left(z b_{\perp}\right)}{s_{1} s_{2}}, \\
& \alpha\left(v_{1}, z_{0}\right) \rightarrow \alpha_{1}=s_{1} \int_{-\infty}^{\infty} d \lambda \varphi\left[z_{3}^{2}+z_{\perp}^{2}+\lambda^{2} ; \rho\right], \\
& \alpha\left(v_{2}, z_{0}\right) \rightarrow \alpha_{2}=s_{2} \int_{-\infty}^{\infty} d \lambda \varphi\left[z_{4}^{2}+(z-b)_{\perp}^{2}+\lambda^{2} ; \rho\right] .
\end{aligned}
$$

The differential cross section of the quark-quark scattering is expressed through the amplitude (10.17) as

$$
\frac{d \sigma_{q q}}{d t} \approx \frac{1}{9} \frac{1}{s^{2}} \sum_{k l} \sum_{m n}\left|T_{l n}^{k m}(s, t)\right|^{2} .
$$

By inserting (10.17) into (10.24) and making analytical continuation to Minkowski space one finds

$$
\frac{d \sigma_{q q}(t)}{d t}=\frac{2}{9} n_{c}^{2}\left[\operatorname{coth}^{2} \chi F_{s}^{2}(t)+\frac{2}{3} \frac{\operatorname{coth} \chi}{\sinh \chi} F_{c}(t) F_{s}(t)+\frac{11}{3} \frac{1}{\sinh ^{2} \chi} F_{c}^{2}(t)\right],
$$

where

$$
F_{s}(t)=\int d^{2} \vec{b}_{\perp} e^{i b_{\perp} q} w_{s}\left(\gamma=\pi / 2, b_{\perp}^{2}\right), \quad F_{c}(t)=\int d^{2} \vec{b}_{\perp} e^{i b_{\perp} q} w_{c}\left(\gamma=\pi / 2, b_{\perp}^{2}\right) .
$$

In the asymptotic limit $(\sinh \chi \sim s, \operatorname{coth} \chi \rightarrow 1)$ the result (10.25) coincides with that one found in [53]

$$
\frac{d \sigma}{d t} \approx \frac{2}{9} n_{c}^{2} F_{s}^{2}(t)
$$

In the weak field limit we reproduce the one-loop single instanton results (10.12) and (10.13).

Considering the quark-antiquark scattering we have to take into account that it is possible to treat an antiquark with velocity $v_{2}$ as a quark moving backward in time with velocity $-v_{2}$. As a result, the scalar product of velocities changes the $\operatorname{sign}\left(v_{1}^{q} v_{2}^{\bar{q}}\right)=-\left(v_{1}^{q} v_{2}^{q}\right)$ and the scattering angles are related as

$$
\chi_{q q} \rightarrow i \pi-\chi_{q \bar{q}} .
$$

Then one gets

$$
\frac{d \sigma_{q \bar{q}}(t)}{d t}=\frac{2}{9} n_{c}^{2}\left[\operatorname{coth}^{2} \chi F_{s}^{2}(t)-\frac{2}{3} \frac{\operatorname{coth} \chi}{\sinh \chi} F_{c}(t) F_{s}(t)+\frac{11}{3} \frac{1}{\sinh ^{2} \chi} F_{c}^{2}(t)\right] .
$$


The second terms in (10.25) and (10.29) corresponding to the contribution of the $C$-odd amplitude has been missed in [53] because there only $S U_{c}(2)$ QCD is considered.

The spin averaged total quark-quark cross section in the instanton-antiinstanton approximation reads

$$
\sigma_{q q} \approx \frac{2}{9} n_{c}^{2} \int_{0}^{\infty} d \boldsymbol{q}_{\perp}^{2}\left[F_{s}^{2}\left(\boldsymbol{q}_{\perp}^{2}\right)+\frac{4}{3} \frac{m^{2}}{s} F_{c}\left(\boldsymbol{q}_{\perp}^{2}\right) F_{s}\left(\boldsymbol{q}_{\perp}^{2}\right)\right],
$$

which is constant in the high energy $s$ limit. It is finite if the constraint instanton solution is used. In Eq. (10.30), the only term corresponding to the $C=+1$ exchange, Pomeron, survives at asymptotic, while the $C=-1$ contribution, odderon, (second term in (10.30) ) is suppressed by the small factor $\sim m^{2} / s$. The growing part of the total cross section arises as

$$
\Delta \sigma_{q q} \sim\left(n_{c} \rho_{c}^{4}\right)^{2} \Delta(t) \ln s,
$$

considering the inelastic quark - quark scattering in the instanton-antiinstanton background [53].

As it was discussed in detail in [16, 53], the model of the Pomeron based on nonperturbative gluon exchanges explains many properties of the diffractive scattering: the effective vector-like exchange $(10.12)$, the additive quark rule and the main features of the total cross section (10.30), (10.31).

It is important to note that the original Wilson loop (10.15) has essentially Minkowskian light-cone geometry whereas the instanton calculations of Wilson loop are performed in the Euclidean QCD. In analogy with the quark form factor the analytical continuation from Minkowski space to Euclidean one and $v$. $v$. becomes possible since the dependence of the Wilson loop on the total energy $s$ and transverse momentum squared $t$ is factorized in (10.12) and (10.22). At high energy, the amplitude is $s$ - independent both in the perturbative and nonperturbative cases. At the same time, the $t$-dependence of the amplitude is naturally expressed through the nonperturbative instanton field. Notice that in the original expression for the Wilson loop (10.15), the nonlocal instanton correlator was integrated over both space-like and time-like separations $x^{2}$ corresponding to the distance between different points on the contour $C_{\mathrm{qq}}$, whereas the final expressions (10.13), (10.27) depend on the space-like variable $t$. Thus we can proceed the formal calculations in Minkowski space and then make the Wick rotation $\boldsymbol{k}_{\perp}^{2} \rightarrow-\boldsymbol{k}_{\perp}^{2}$, or, that is more natural from the point of view the instanton model, to perform formal manipulations in the Euclidean space and than make analytical continuation.

\section{Conclusions}

Besides the considerable progress in investigation of the role of nonperturbative QCD vacuum structure (in particular, of the instanton phenomena) in low and moderate energy domains of hadronic physics, nowadays there is a lack of understanding of their role in high energy processes which are intensively studied in modern experiments in particle physics. In this work we presented the results of analysis of the structure of nonperturbative corrections 
in such important quantities as the quark form factor and the cross section of the diffractive quark-quark scattering at high energy. The quark scattering process was considered in background of QCD vacuum which is described within the instanton liquid model. The instanton contribution to the electromagnetic quark form factor is calculated in the large momentum transfer regime. We estimated analytically the weak field approximation for the instanton contribution and find the all-order result in the asymptotic regime. The latter was performed considering the Gaussian simulation of the constraint instanton profile function. We found that the leading contribution to high energy asymptotic behavior is provided by the lowest order terms. Although the latter result could be treated as an argument in favor of validity of the weak field approximation, the better work has to be done in this direction since the results for Gaussian profile and the instanton in the singular gauge may be different in general.

The instanton effects are more important for phenomenology of the hadronic processes possessing two different energy scales. (For more detailed discussions see the works [11]). One of such situations - quark-quark diffractive scattering — was considered in the last Section of the present work. This approach supports the model of the Pomeron as exchange by nonperturbative gluons interpolated by the classical instanton field. We have shown that the $C$-odd (odderon) amplitude is suppressed as $1 / s$ compared to the $C$-even (Pomeron) one. In the case of diffractive scattering, the total center-of-mass energy $s$ (hard characteristic scale) is large while the squared momentum transfer $-t$ is small compared to the latter: $-t \ll s$, but nevertheless larger than any IR scale. Besides this, the other cases of interest where the nonperturbative (including instanton induced) effects may be significant are the saturation in deep-inelastic scattering at small-x [73], and the transverse momentum distribution of vector bosons in the Drell-Yan process 48]. The latter is one of the most important objects of the experimental investigations (in particular, in the context of searches for New Physics and Higgs bosons - at future LHC and Tevatron experiments [74]), as well as theoretical studies of the predictive power of pQCD at various energy scales and the role of nonperturbative physics (see, e.g., 75] and references therein).

\section{Acknowledgements}

The useful discussions on various aspects of this work and critical comments by B.I. Ermolaev, N.I. Kochelev, E.A. Kuraev, L. Magnea, S.V. Mikhailov, N.G. Stefanis, and O.V. Teryaev are thanked. The work is partially supported by RFBR (Grant nos. 04-02-16445, 03-02-17291, 02-02-16194), Russian Federation President's Grant no. 1450-2003-2, and INTAS (Grant no. 00-00-366).

\section{Appendix}

The evaluation of the path integrals in the weak-field (one-loop) approximation was performed by using the following expressions: The partial derivatives in $n$-dimensional spacetime are presented as

$$
\partial_{z}^{2}=2 n \partial_{z^{2}}+4 z^{2} \partial_{z^{2}}^{2}, \quad \partial_{\mu} \partial_{\nu}=2 g_{\mu \nu} \partial_{z^{2}}+4 z_{\mu} z_{\nu} \partial_{z^{2}}^{2}
$$


The scalar products of the scattering vectors (9.6) in terms of the scattering angle $\chi$ read

$$
\begin{gathered}
v_{\mu}^{1} v_{\nu}^{2} z_{\mu} z_{\nu}=z^{2} \cosh \chi+\sigma \tau \sinh ^{2} \chi \\
z^{2}=\left(v_{1} \sigma+v_{2} \tau\right)^{2}=\sigma^{2}+\tau^{2}+2 \sigma \tau \cosh \chi .
\end{gathered}
$$

To extract the universal cusp factor, we use the integrals:

$$
\int_{0}^{\infty} d \sigma \int_{0}^{\infty} d \tau \exp \left[-\alpha\left(\sigma^{2}+\tau^{2}+2 \sigma \tau \cosh \chi\right)\right]=\frac{1}{2 \alpha} \frac{\chi}{\sinh \chi}
$$

and

$$
\int_{0}^{\infty} d \sigma \int_{0}^{\infty} d \tau \sigma \tau \exp \left[-\alpha\left(\sigma^{2}+\tau^{2}+2 \sigma \tau \cosh \chi\right)\right]=-\frac{1-\chi \operatorname{coth} \chi}{4 \alpha^{2} \sinh ^{2} \chi}
$$

Therefore, for arbitrary functions $\Delta_{i}\left(z^{2}\right)$ we get:

$$
\Delta_{i}^{\{k\}}\left(z^{2}\right)=(-)^{k} \int_{0}^{\infty} d \alpha \alpha^{k} \mathrm{e}^{-\alpha z^{2}} \bar{\Delta}_{i}(\alpha)
$$

and gets

$$
\begin{gathered}
\int_{0}^{\infty} d \sigma \int_{0}^{\infty} d \tau \Delta^{\prime}\left(z^{2}\right)=-\frac{\chi}{2 \sinh \chi} \Delta(0) \\
\int_{0}^{\infty} d \sigma \int_{0}^{\infty} d \tau z^{2} \Delta^{\prime \prime}\left(z^{2}\right)=\frac{\chi}{2 \sinh \chi} \Delta(0), \\
\int_{0}^{\infty} d \sigma \int_{0}^{\infty} d \tau \sigma \tau \Delta^{\prime \prime}\left(z^{2}\right)=-\frac{1-\chi \operatorname{coth} \chi}{4 \sinh ^{2} \chi} \Delta(0) .
\end{gathered}
$$

In the all-order instanton calculations, the Gaussian integrals over $z_{0}$ are taken by

$$
\begin{gathered}
\int d^{2} z_{\perp}\left(z_{\perp}^{2}\right)^{n} e^{-\alpha z_{\perp}^{2}}=n ! \frac{\pi}{\alpha^{n+1}} \\
\int_{-\infty}^{\infty} d y y^{n} e^{-p y^{2}-q y}=\sqrt{\frac{\pi}{p}} e^{\frac{q^{2}}{4 p}} \sum_{s=0}^{[n / 2]} \bar{C}_{s}^{n}\left(-\frac{q}{2 p}\right)^{n-2 s} \frac{1}{p^{s}}
\end{gathered}
$$

where

$$
\bar{C}_{s}^{n}=\frac{n !}{s !(n-2 s) ! 2^{2 s}}
$$

and we use the binomial formula

$$
\left(z_{3}^{2}+z_{\perp}^{2}\right)^{n}=\sum_{k=0}^{n} C_{k}^{n} z_{\perp}^{2 k} z_{3}^{2(n-k)}
$$




\section{References}

[1] R. Brock, et. al. [CTEQ Collaboration], Rev. Mod. Phys. 67 (1995) 157.

[2] Yu. Dokshitzer, D. Dyakonov, S. Troyan, Phys. Reports 58 (1980) 269; A.H. Mueller, Phys. Reports 73 (1981) 237.

[3] R. Petronzio, S. Simula, G. Ricco, Phys. Rev. D67 (2003) 094004; S. Simula, Phys. Lett. B 574 (2003) 189.

[4] M. Osipenko et al. [CLAS Collaboration], Phys. Rev. D67 (2003) 092001; M. Osipenko et al., arXiv:hep-ex/0309052.

[5] O. Nachtmann, Nucl. Phys. B63 (1973) 237.

[6] E. Shuryak, Nucl. Phys. B203 (1982) 93, 116, 140.

[7] D. Diakonov, V. Petrov, Nucl. Phys. B245 (1984) 259.

[8] T. Schäfer, E.V. Shuryak, Rev. Mod. Phys. 70 (1998) 323.

[9] A.E. Dorokhov, Y.A. Zubov, N.I. Kochelev, Sov. J. Part. Nucl. 23 (1992) 522.

[10] A. Hasenfratz, C. Nieter, Phys. Lett. B439 (1998) 366.

[11] A.E. Dorokhov, L. Tomio, Phys. Rev. D62 (2000) 014016; I.V. Anikin, A.E. Dorokhov, L. Tomio, Phys. Part. Nucl. 31 (2000) 509.

[12] A.E. Dorokhov, JETP Lett. 77 (2003) 63 [Pisma Zh. Eksp. Teor. Fiz. 77, 68 (2003)].

[13] A.E. Dorokhov, W. Broniowski, Eur. Phys. J. C32(2003)79.

[14] F.E. Low, Phys. Rev. D12 (1975) 163.

[15] S. Nussinov, Phys. Rev. Lett. 34 (1975) 1286.

[16] P.V. Landshoff, O. Nachtmann, Z. Phys. C35 (1987) 405.

[17] A. Bassetto, M. Ciafaloni, G. Marchesini, Phys. Reports 100 (1983) 201; O. Nachtmann, Ann. Phys. (N.Y.) 209 (1991) 436.

[18] G. Korchemsky, Phys. Lett. B325 (1994) 459; I. Korchemskaya, Nucl. Phys. B490 (1997) 306.

[19] Yu.M. Makeenko, A.A. Migdal, Phys. Lett. B88 (1979) 135; Nucl. Phys. B188 (1981) 269.

[20] V.S. Dotsenko, S.N. Vergeles, Nucl. Phys. B169 (1980) 527; R.A. Brandt, F. Neri, M.-A. Sato, Phys. Rev. D24 (1981) 879; R.A. Brandt, A. Gocksch, M.A. Sato, F. Neri, Phys. Rev. D26 (1982) 3611.

[21] A.V. Belitsky, A.S. Gorsky, G.P. Korchemsky, Nucl. Phys. B667 (2003) 3.

[22] A.I. Karanikas, C.N. Ktorides, N.G. Stefanis, Eur. Phys. J. C26 (2003) 445.

[23] G. Parisi, Phys. Lett. B90 (1980) 295; G. Curci, M. Greco, Phys. Lett. B92 (1980) 175.

[24] L. Magnea, G. Sterman, Phys. Rev. D42 (1990) 4222; L. Magnea, Nucl. Phys. B593 (2001) 269.

[25] B.I. Ermolaev, S.I. Troyan, Nucl. Phys. B590 (2000) 521.

[26] N.I. Kochelev, Phys. Lett. B565 (2003) 131. 
[27] V.V. Sudakov, Sov. Phys. JETP 3 (1956) 65; [Zh. Eksp. Teor. Fiz. 30 (1956) 87].

[28] R. Barbieri, J.A. Mignaco, E. Remiddi, Nuovo Cim. A11 (1972) 824, 865; P. Mastrolia, E. Remiddi, Nucl. Phys. B664 (2003) 341; R. Bonciani, P. Mastrolia, E. Remiddi, Nucl. Phys. B676 (2004) 399;

[29] R. Jackiw, Ann. Phys. (NY) 48 (1968) 292; P.M. Fishbane, J.D. Sullivan, Phys. Rev. D4 (1971) 458.

[30] J.M. Cornwall, G.Tiktopoulos, Phys. Rev. D13 (1976) 3370; J. Frenkel, J.C. Taylor, Nucl. Phys. B116 (1976) 185; E.C. Poggio, G. Pollack, Phys. Lett. B71 (1977) 135.

[31] E.A. Kuraev, V.S. Fadin, Yad. Fiz. 27 (1978) 1107.

[32] B.I. Ermolaev, V.S. Fadin, JETP Lett. 33 (1981) 269; [Pisma Zh. Eksp. Teor. Fiz. 33 (1981) 285]; V.S. Fadin, Sov. J. Nucl. Phys. 37 (1983) 2145; [Yad. Fiz. 37 (1983) 408]; B.I. Ermolaev, V.S. Fadin, L.N. Lipatov, Sov. J. Nucl. Phys. 45 (1987) 508; [Yad. Fiz. 45 (1987) 817].

[33] V.V. Belokurov, N.I. Usseyukina, Phys. Lett. B94 (1980) 251; H.D. Dahmen, F. Steiner, Z. Phys. C11 (1981) 247.

[34] J.C. Collins, Adv. Ser. Direct. High Energy Phys. 5 (1989) 573; arXiv:hep-ph/0312336.

[35] A.H. Mueller, Phys. Rev. D20 (1979) 2037; J.C. Collins, Phys. Rev. D22 (1980) 1478.

[36] A. Sen, Phys. Rev. D24 (1981) 3281.

[37] G. Korchemsky, A. Radyushkin, Sov. J. Nucl. Phys. 45 (1987) 910; G. Korchemsky, A. Radyushkin, Nucl. Phys. B283 (1987) 342.

[38] G. Korchemsky, Phys. Lett. B217 (1989) 330.

[39] G. Korchemsky, Phys. Lett. B220 (1989) 629.

[40] J.G.M. Gatheral, Phys. Lett. B133 (1983) 90; J. Frenkel, J.C. Taylor, Nucl. Phys. B246 (1984) 231.

[41] G.C. Gellas, A.I. Karanikas, C.N. Ktorides, N.G. Stefanis, Phys. Lett. B412 (1997) 95.

[42] M. Beneke, Phys. Reports 317 (1999) 1; M. Beneke, V.M. Braun, hep-ph/0010208.

[43] D.V. Shirkov, I.L. Solovtsov, Phys. Rev. Lett. 79 (1997) 1209; D.V. Shirkov, I.L. Solovtsov, hep-ph/9604363.

[44] A. Polyakov, Phys. Lett. B82 (1979) 247; Nucl. Phys. B164 (1980) 171; Gauge Fields and Strings, Harwood, 1987

[45] I. Korchemskaya, G. Korchemsky, Phys. Lett. B287 (1992) 169;

[46] A.E. Dorokhov, I.O. Cherednikov, Phys. Rev. D66 (2002) 074009; Eur. Phys. J. A18 (2003) 215.

[47] A.E. Dorokhov, I.O. Cherednikov, Phys. Rev. D67 (2003) 114017.

[48] G. Korchemsky, G. Sterman, Nucl. Phys. B437 (1995) 415; M. Beneke, V.M. Braun, Nucl. Phys. B454 (1995) 253.

[49] S.V. Mikhailov, Phys. Lett. B416 (1998) 421.

[50] N.G. Stefanis, W. Schroers, H.C. Kim, Eur. Phys. J.C18 (2000) 137. 
[51] N. Andrei, D. Gross, Phys. Rev. D18 (1978) 468; L. Baulieu, J. Ellis, M. Gaillard, W. Zakrzewski, Phys. Lett. B77 (1978) 290.

[52] I.I. Balitsky, Phys. Lett. B273 (1991) 282; V.I. Zakharov, Nucl. Phys. B385 (1992) 452; I.I. Balitsky, V.M. Braun, Phys. Lett. B314 (1993) 237.

[53] E. Shuryak, I. Zahed, Phys. Rev. D62 (2000) 085014; M. Nowak, E. Shuryak, I. Zahed, Phys. Rev. D64 (2001) 034008.

[54] D. Kharzeev, Y. Kovchegov, E. Levin, Nucl. Phys. A690 (2001) 621.

[55] S. Moch, A. Ringwald, F. Schrempp, Nucl. Phys. B507 (1997) 134.

[56] F. Schrempp, A. Utermann, Phys. Lett. B543 (2002) 197.

[57] A. Ringwald, Nucl. Phys. Proc. Suppl. 121 (2003) 145, hep-ph/0210209.

[58] A.E. Dorokhov, hep-ph/0302242

[59] I.O. Cherednikov, Surv. High Energy Phys. 18 (2003) 205; hep-ph/0305055.

[60] A.E. Dorokhov, N.I. Kochelev, Y.A. Zubov, Int. J. Mod. Phys. A8 (1993) 603.

[61] E. Meggiolaro, Phys. Rev. D53 (1996) 3835.

[62] M.A. Shifman, A.I. Vainshtein, V.I. Zakharov, Nucl. Phys. B163 (1980) 46.

[63] S.V. Ivanov, G.P. Korchemsky, A.V. Radyushkin, Yad. Fiz. 44 (1986) 230 [Sov. J. Nucl. Phys. 44 (1986) 145]; S.V. Ivanov, G.P. Korchemsky, Phys. Lett. B154 (1985) 197.

[64] C. G. Callan, R. Dashen, D. J. Gross, Phys. Rev. D17 (1978) 2717.

[65] G. 't Hooft, Phys. Rev. D14 (1976) 3432.

[66] A.E. Dorokhov, S.V. Esaibegyan, S.V. Mikhailov, Phys. Rev. D56 (1997) 4062;

[67] A.E. Dorokhov, S.V. Esaibegyan, A. E. Maximov, S.V. Mikhailov, Eur. Phys. J. C13 (2000) 331.

[68] E.V. Shuryak, hep-ph/9909458.

[69] T.R. Morris, D.A. Ross, C.T. Sachrajda, Nucl. Phys. B255 (1985) 115.

[70] D.A. Smith, M.J. Teper [UKQCD collaboration], Phys. Rev. D58 (1998) 014505.

[71] F. Schrempp, J. Phys. G28 (2002) 915.

[72] M. D’Elia, A. Di Giacomo, E. Meggiolaro, Phys. Lett. B408 (1997) 315.

[73] F. Schrempp, A. Utermann, hep-ph/0301177.

[74] S. Catani et al., hep-ph/0005025; S. Catani, hep-ph/0005233; S. Catani, D. de Florian, M. Grazzini, JHEP 0201 (2002) 015; G. Bozzi, S. Catani, D. de Florian, M. Grazzini, Phys. Lett. B564 (2003) 65.

[75] J.-w. Qiu, X.-f. Zhang, Phys. Rev. D63 (2001) 114011. 


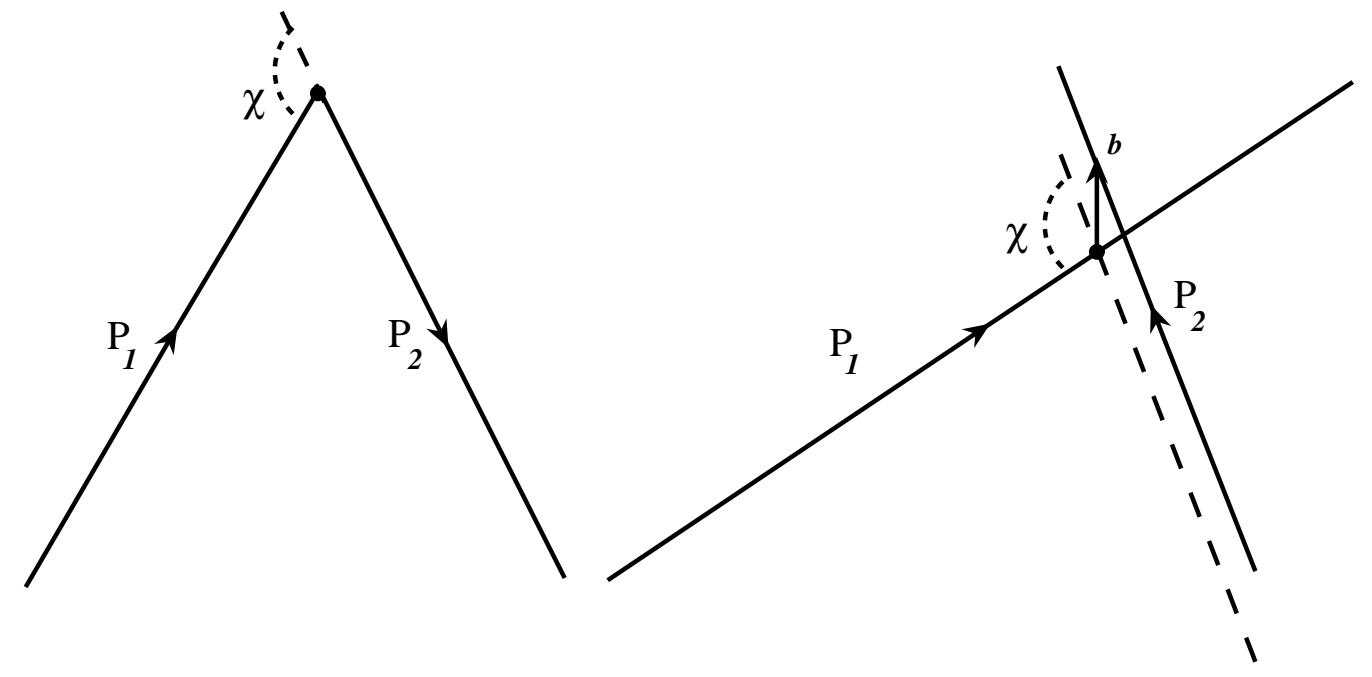

(a)

(b)

Figure 1: The notations of the quark momenta for the (a) quark form factor, and (b) quark-quark scattering. 


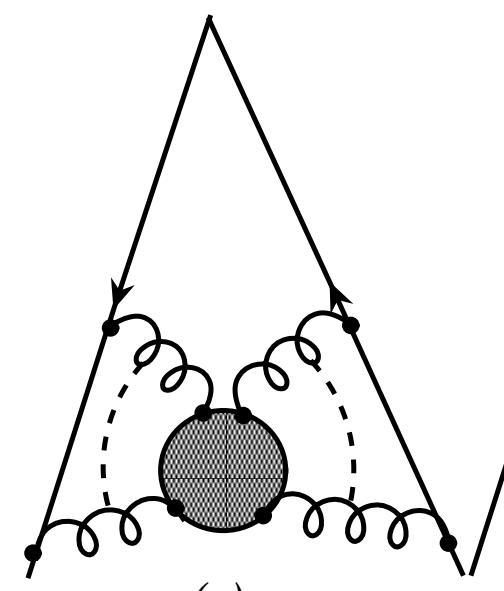

(a)

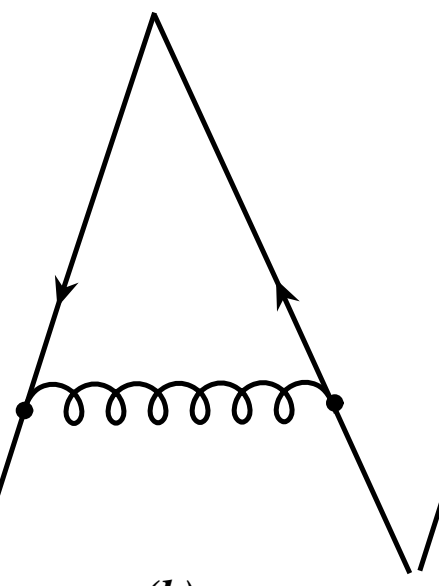

(b)

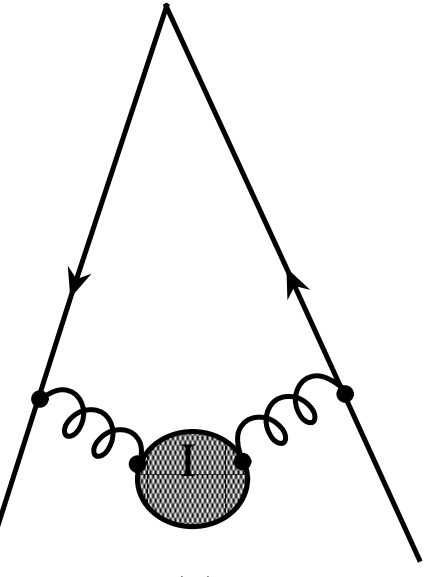

(c)

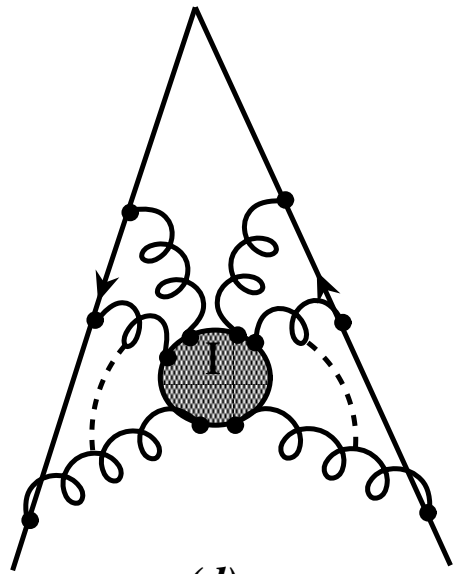

(d)

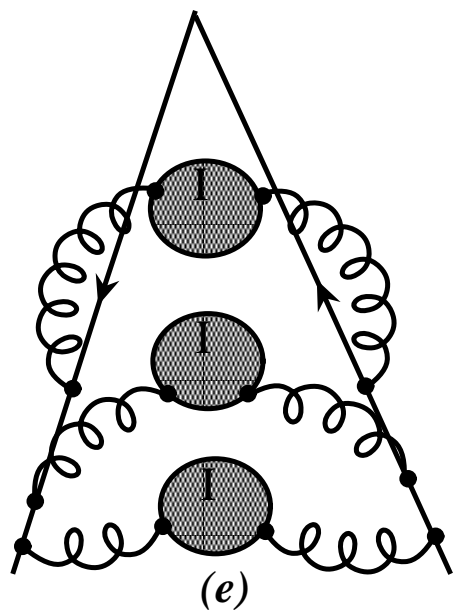

(e)

Figure 2: The total cusp-dependent part of the Wilson loop integral for the quark form factor (a); the leading order contributions of the perturbative (b) and nonperturbative (single-instanton) (c) fields; (d) the all-order single instanton result; (e) the exponentiation of the single instanton result. 


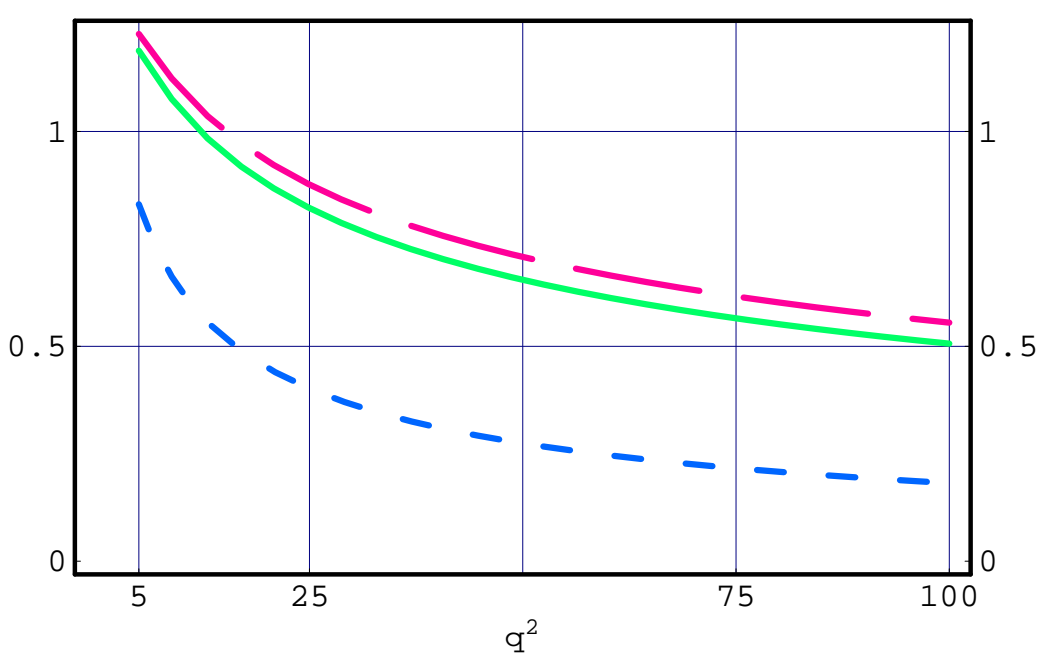

Figure 3: The asymptotic behaviour of the quark form factor is shown as the function of the dimensionless variable $q^{2}=Q^{2} / \Lambda^{2}$, up to terms $O\left(\ln \ln q^{2}\right)$. The long-dash presents the contribution of one loop perturbative terms; the solid line represents the total form factor including the instanton induced part, Eq. (8.21). For comparison, the leading $\left(\sim \ln q^{2} \ln \ln q^{2}\right)$ perturbative contribution is shown separately - the short-dash line.

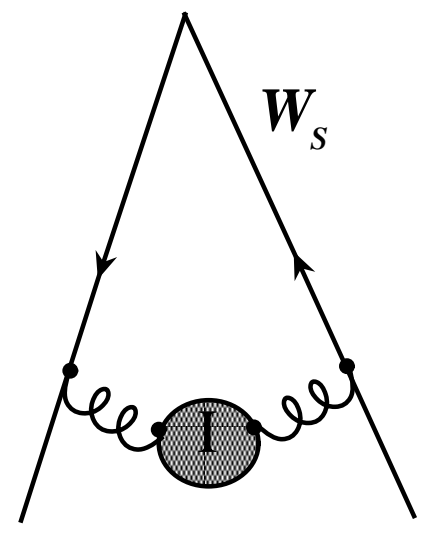

(a)

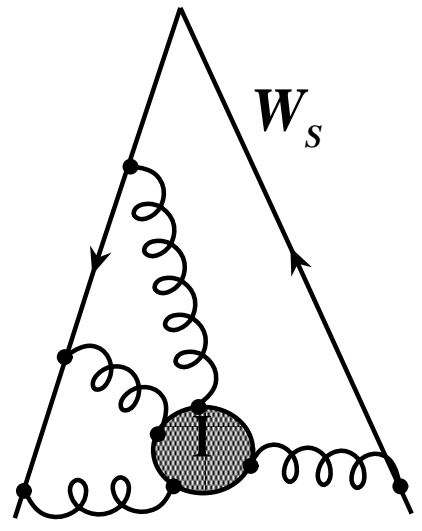

(b)

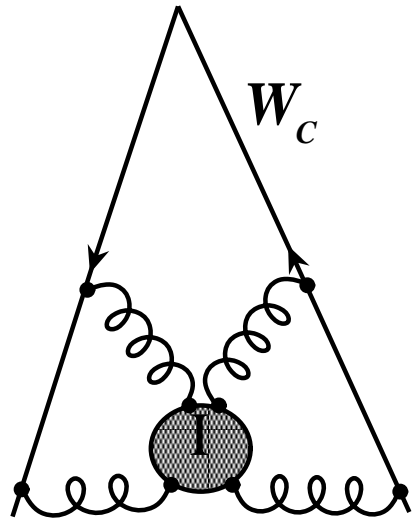

(c)

Figure 4: Schematic representation of the lowest order instanton contributions. 


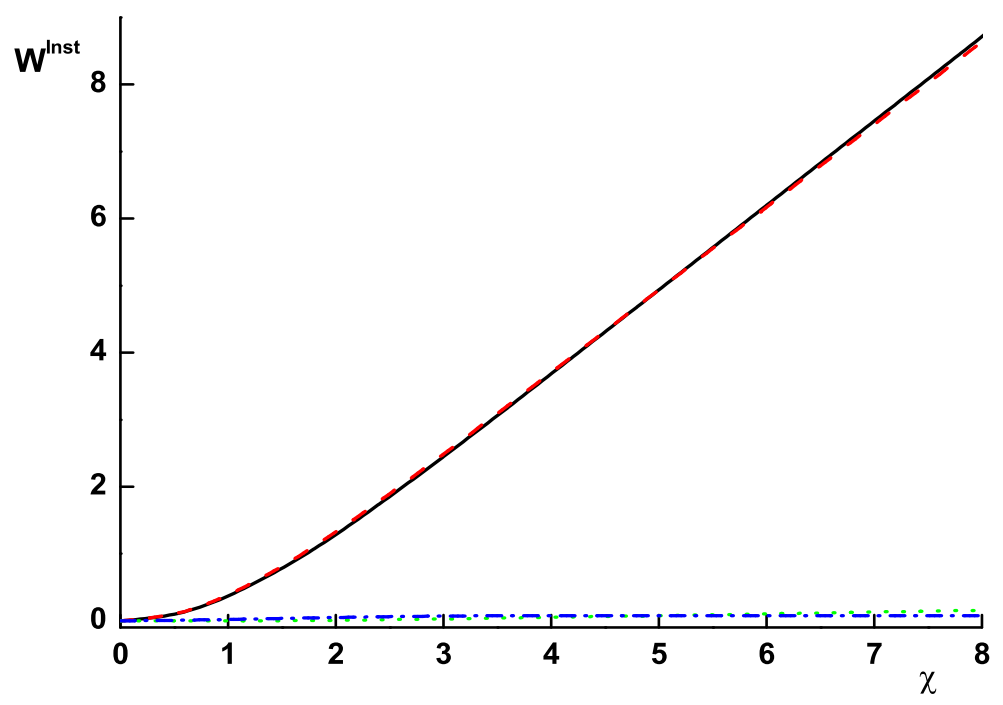

Figure 5: Lowest orders instanton contributions to the Wilson integral with spin factors. The leading term $\left\langle\alpha_{1}^{1} \alpha_{2}^{1}\right\rangle(\gamma)$ is the dashed line, next-to-leading terms $\left\langle\alpha_{1}^{1} \alpha_{2}^{3}\right\rangle(\gamma)$ and $\left\langle\alpha_{1}^{2} \alpha_{2}^{2}\right\rangle(\gamma)$ are the dotted and dash-dotted line, correspondingly. The sum of these contributions is the solid line.

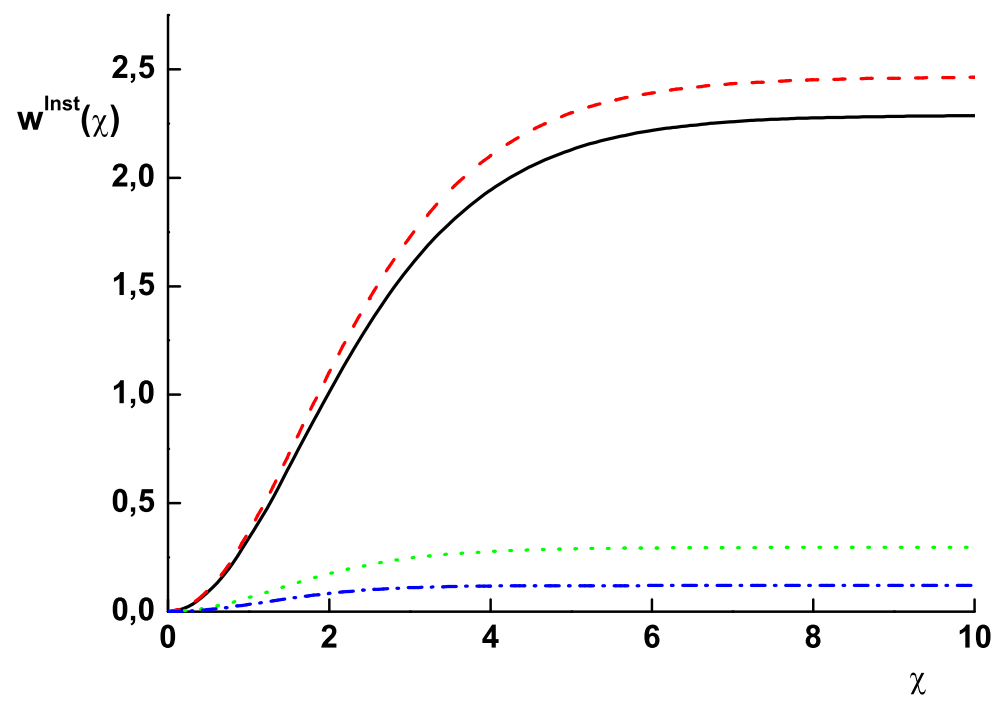

Figure 6: Lowest orders instanton contributions to the Wilson integral without spin factors. The leading term $\left\langle\alpha_{1}^{1} \alpha_{2}^{1}\right\rangle(\gamma)$ is dashed line, next to leading terms $\left\langle\alpha_{1}^{1} \alpha_{2}^{3}\right\rangle(\gamma)$ and $\left\langle\alpha_{1}^{2} \alpha_{2}^{2}\right\rangle(\gamma)$ are dotted and dash-dotted line, correspondingly. The sum of these contributions is the solid line. 


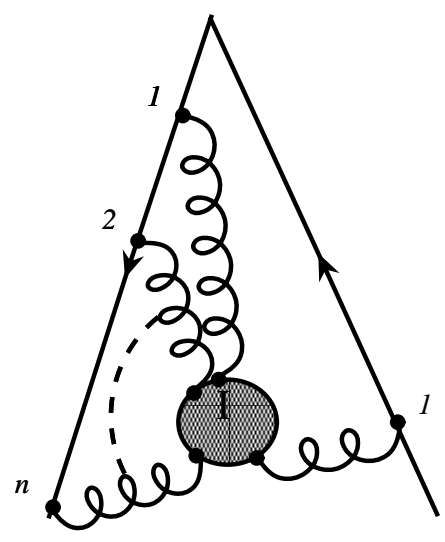

(a)

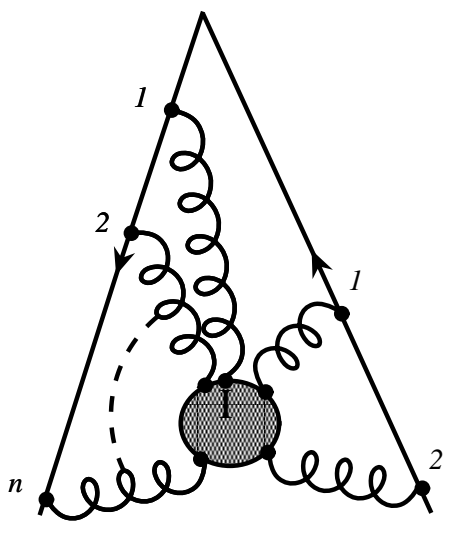

(b)

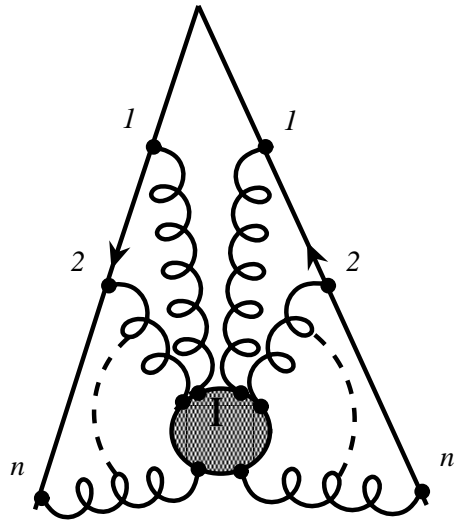

(c)

Figure 7: Schematic representation of partial summation of the instanton contributions. 

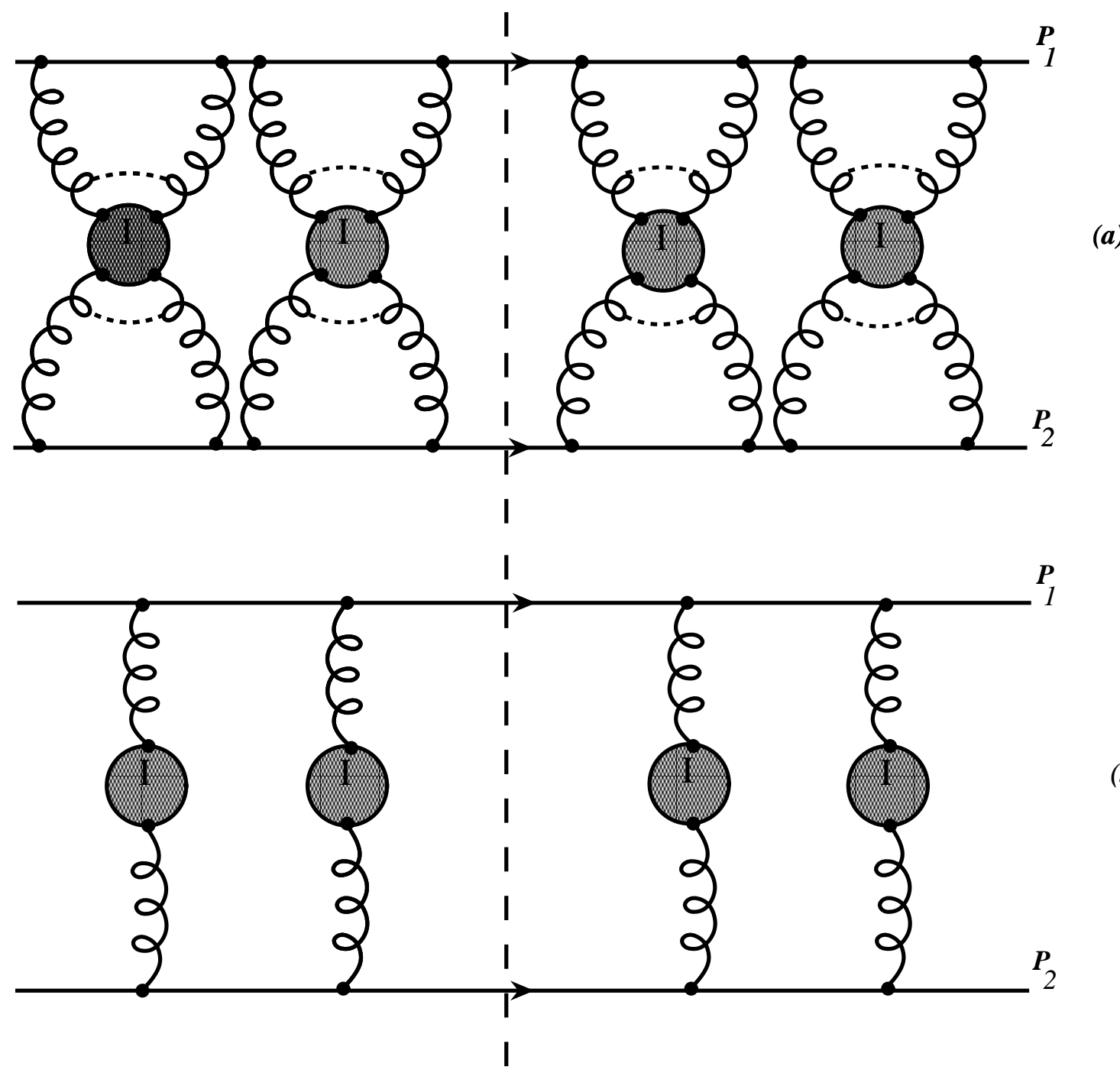

(b)

Figure 8: Schematic representation of partial summation of the instanton contributions to the quark-quark scattering. 


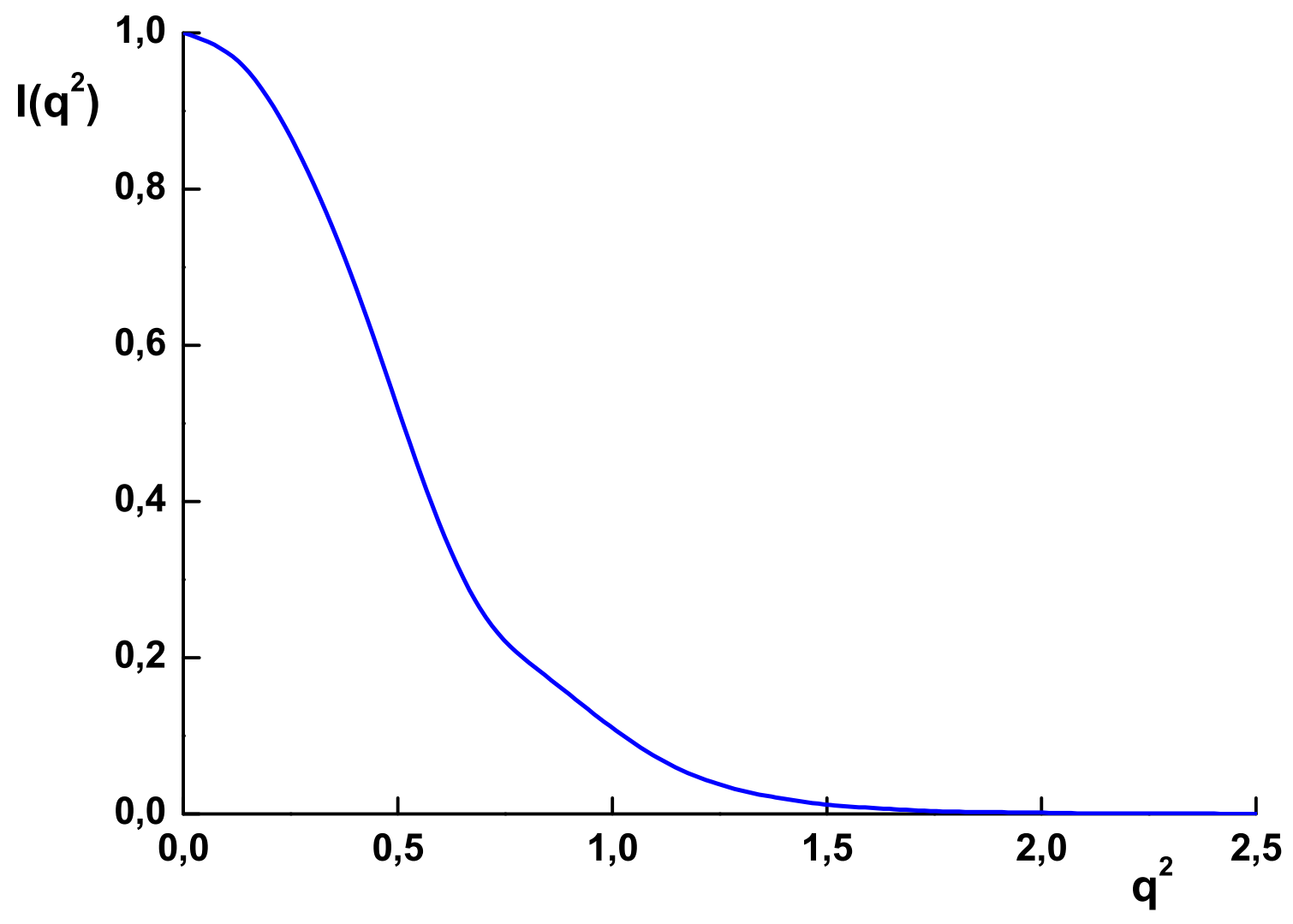

Figure 9: Normalized quark-quark scattering amplitude, Eq. (10.13). 